Review

\title{
Infrared protein crystallography
}

\author{
J. Timothy Sage a,*, Yunbin Zhang a , John McGeehan ${ }^{\text {c,e }}$, Raimond B.G. Ravelli c,f \\ Martin Weik ${ }^{\mathrm{d}, \mathrm{g}, \mathrm{h}, \mathrm{i}}$, Jasper J. van Thor ${ }^{\mathrm{b}, * *}$ \\ a Department of Physics and Center for Interdisciplinary Research on Complex Systems, Northeastern University, Boston, MA 02115, USA \\ b Division of Molecular Biosciences, Imperial College London, London SW7 2AZ, UK \\ c EMBL Grenoble, 6 rue Jules Horowitz, 38042 Grenoble, France \\ d Commissariat à l'Energie Atomique, Institut de Biologie Structurale, F-38054 Grenoble, France \\ e Biophysics Laboratories, Institute of Biomedical and Biomolecular Sciences, School of Biological Sciences, University of Portsmouth, PO1 2DY, UK \\ ${ }^{\mathrm{f}}$ Department of Molecular Cell Biology, Leiden University Medical Center, P.O. Box 9600, 2300RC Leiden, The Netherlands \\ g CNRS, F-38027 Grenoble, France \\ h Université Joseph Fourier, F-38000, Grenoble, France \\ ${ }^{\mathrm{i}}$ ESRF, 6 rue Jules Horowitz, BP 220, 38043 Grenoble Cedex, France
}

\section{A R T I C L E I N F O}

\section{Article history:}

Received 30 November 2010

Received in revised form 22 February 2011

Accepted 23 February 2011

Available online 2 March 2011

\section{Keywords:}

Infrared crystallography

Infrared dichroism

Radiation damage

Vibrational spectroscopy

\begin{abstract}
A B S T R A C T
We consider the application of infrared spectroscopy to protein crystals, with particular emphasis on exploiting molecular orientation through polarization measurements on oriented single crystals. Infrared microscopes enable transmission measurements on individual crystals using either thermal or nonthermal sources, and can accommodate flow cells, used to measure spectral changes induced by exposure to soluble ligands, and cryostreams, used for measurements of flash-cooled crystals. Comparison of unpolarized infrared measurements on crystals and solutions probes the effects of crystallization and can enhance the value of the structural models refined from X-ray diffraction data by establishing solution conditions under which they are most relevant. Results on several proteins are consistent with similar equilibrium conformational distributions in crystal and solutions. However, the rates of conformational change are often perturbed. Infrared measurements also detect products generated by X-ray exposure, including $\mathrm{CO}_{2}$. Crystals with favorable symmetry exhibit infrared dichroism that enhances the synergy with X-ray crystallography. Polarized infrared measurements on crystals can distinguish spectral contributions from chemically similar sites, identify hydrogen bonding partners, and, in opportune situations, determine three-dimensional orientations of molecular groups. This article is part of a Special Issue entitled: Protein Structure and Function in the Crystalline State.
\end{abstract}

(c) 2011 Elsevier B.V. All rights reserved.

\section{Introduction}

Crystallography has become so strongly associated with X-ray diffraction that the two terms are nearly synonymous in the minds of many practicing scientists. However, it is worth recalling that crystallographers were making important contributions to the understanding of matter long before Röntgen's discovery of X-rays in 1897. In the 19 th century, for example, crystallographers were leading proponents of the atomic theory of matter, because it allowed successful quantitative explanations of many crystal shapes. Optical crystallog-

\footnotetext{
i This article is part of a Special Issue entitled: Protein Structure and Function in the Crystalline State.

* Corresponding author. Tel.: +1 617373 2918; fax: +1 6173732943.

** Corresponding author.

E-mail addresses: jtsage@neu.edu (J.T. Sage), j.vanthor@imperial.ac.uk (J.J. van Thor).
}

raphy, over a growing frequency range from infrared to X-ray, continues to provide molecular insights.

Interest in spectroscopic measurements in the crystalline phase is increasing [1-8]. This is largely driven by the desire to establish that the detailed three-dimensional structural models derived from X-ray diffraction on protein crystals actually coincide with the structures of the proteins in solution. In addition to the possibility that intermolecular interactions in the crystalline environment may perturb the structure, there is a growing realization that intense X-radiation from synchrotron sources modifies the molecules under study [9-12]. This is especially a concern with regard to accurate structural description of metalloprotein active sites, where the redox activity that is integral to their biological function also renders them more susceptible to structural changes by trapping photoelectrons [13-19]. Single crystal spectroscopy can also identify protein intermediate states in kinetic crystallography, which aims at their generation, trapping, and structural characterization [20]. Unique features of the crystalline environment, most notably the high degree of molecular orientation, 


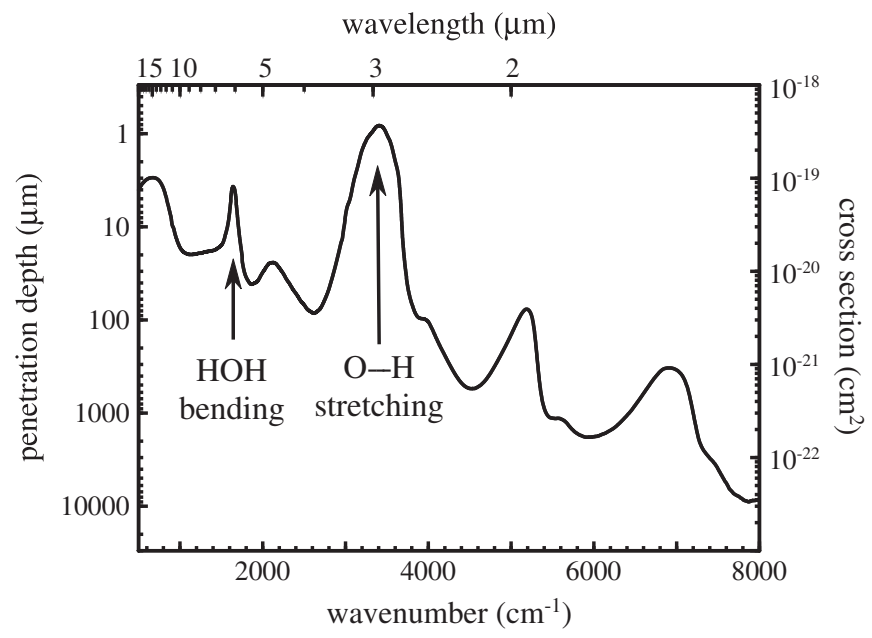

Fig. 1. Solvent absorption governs experimental design for infrared spectroscopy on protein crystals. The frequency-dependent penetration depth $\delta$ and absorption cross section $\sigma$ of pure water are determined from published optical constants [110] as tabulated at http://omlc.ogi.edu/spectra/water/data/wieliczka89.dat.

also create opportunities to obtain spectroscopic information that is not available from measurements on solutions.

Crystalline spectroscopy has emphasized electronic spectroscopy. However, vibrational spectroscopy provides a number of advantages because of its higher information content. This article focuses on infrared measurements on protein crystals-experimental methodology and applications.

\section{Infrared instrumentation}

Biomolecular structure and function depend essentially on water, and solvent absorption (Fig. 1) is the primary practical issue governing infrared instrumentation for any biological application, including measurements on protein crystals. Solvent absorption interferes with any other spectroscopic signal of interest and presents a particularly severe challenge for frequencies near $3400 \mathrm{~cm}^{-1}$, where strong absorption by the symmetric and antisymmetric $\mathrm{O}-\mathrm{H}$ stretching vibrations reduces the penetration depth to approximately $1 \mu \mathrm{m}$ for bulk water at ambient temperature. The penetration depth also drops below $5 \mu \mathrm{m}$ near the $1640 \mathrm{~cm}^{-1} \mathrm{HOH}$ bending vibration. In the condensed phase, intermolecular vibrations give rise to an additional broad absorption feature, peaked near $700 \mathrm{~cm}^{-1}$ in liquid water, as well as strong absorption in the $\mathrm{THz}$ regime below about $150 \mathrm{~cm}^{-1}$. When these regions are of particular interest, solvent replacement with $\mathrm{D}_{2} \mathrm{O}$ can improve sample transmission and increase experimental signal. Nevertheless, practical infrared transmission measurements below $4000 \mathrm{~cm}^{-1}$ on aqueous solutions, which includes nearly all situations of interest for vibrational spectroscopic investigations of biomolecules, require sample thicknesses in the $1-100 \mu \mathrm{m}$ range.

Protein crystals offer both challenges and opportunities for infrared measurements. One significant advantage is that the fractional contribution of protein absorption to the total signal is close to the achievable limit. Molecular alignment also provides spectroscopic information not available from solution measurements for favorable crystal symmetries that allow polarized absorption measurements. The thicknesses of protein crystals often lie in the $1-100 \mu \mathrm{m}$ range suitable for infrared transmission measurements.

However, the lateral dimensions are rarely (if ever) large enough to fill the infrared beam on a commercial FTIR instrument, which is typically on the order of $10 \mathrm{~mm}$. The need to collect infrared radiation and focus to a small area is the primary factor that distinguishes measurements on protein crystals from other infrared spectroscopic studies of biomole- cules. Let us consider the instrumental aspects of infrared spectroscopy in the light of practical constraints on crystal dimensions.

\subsection{Detectors}

Photoconductive mercury-cadmium-telluride (MCT) detectors are presently the most commonly used for biomolecular infrared spectroscopy, and have also been the primary choice for measurements on crystals. They offer spectral coverage at frequencies greater than $1000 \mathrm{~cm}^{-1}$ with higher sensitivity than thermal detectors, but require liquid nitrogen in order to cool the semiconductor detector element, minimizing background signal caused by thermal excitation of charge carriers into the conduction band. Commercial FTIR instruments typically employ single-element detectors, but MCT arrays have become an affordable option for dispersive detection.

Less common detector options include $\mathrm{Cu}$-doped germanium $(\mathrm{Cu}$ : $\mathrm{Ge}$ ), which offers expanded spectral coverage but require cooling with liquid helium. The spectra in Fig. 4 and the lower panel of Fig. 2 were recorded on $\mathrm{Cu}: \mathrm{Ge}$ detectors, which are often available at infrared beamlines at synchrotron sources. Indium antimonide detectors are rarely used in biomolecular spectroscopy because spectral coverage does not extend below about $1800 \mathrm{~cm}^{-1}$. However, they offer slightly higher sensitivity than MCT detectors and are worth considering if samples thinner than about $20-30 \mu \mathrm{m}$ are not available or if higher frequencies are of primary interest.

\subsection{Sources}

Commercial FTIR instruments use infrared radiation emitted by a hot filament, with a spectrum closely approximated by a black body

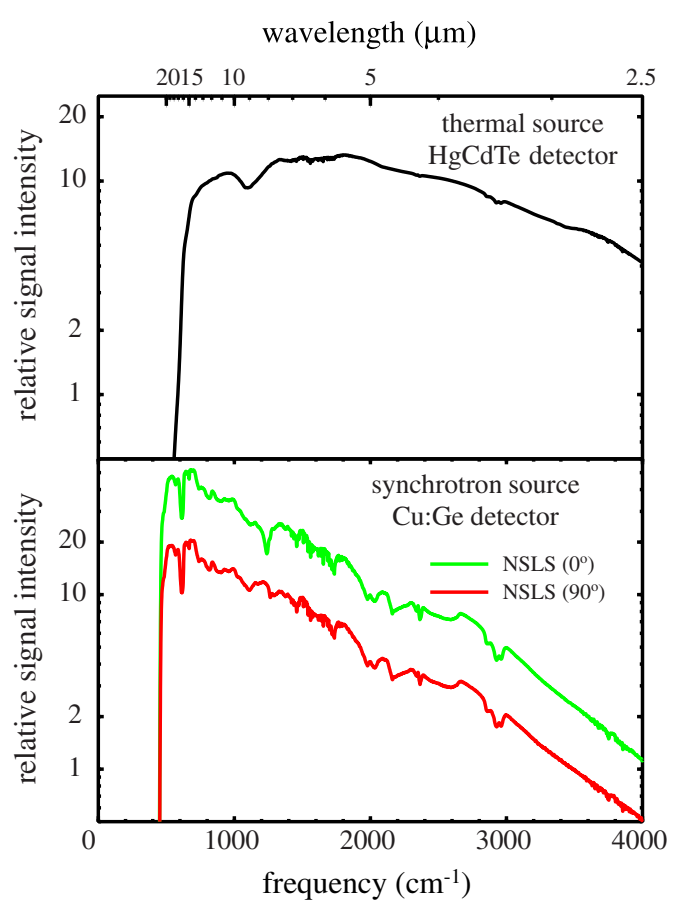

Fig. 2. Infrared spectra measured using thermal (top) and synchrotron (bottom) sources in the absence of sample. The spectrum in the upper panel was recorded on a commercial FTIR spectrometer (Bio-rad FTS-60A), using an MCT detector cooled with liquid nitrogen. Spectra in the lower panel were recorded for two orthogonal orientations of an infrared polarizer placed in the beam at beamline U10A at the National Synchrotron Light Source at Brookhaven National Lab, using a copper-doped germanium detector. The infrared beam derived from the synchrotron bending magnet and delivered to the experimental station exhibits significant intrinsic polarization, while measurements on the laboratory instrument reveal only a weak residual polarization. All data represent the available experimental signal; no attempt has been made to correct for wavelength dependence in the optical path or detector response. 
distribution. These thermal sources are inexpensive and usually quite effective when coupled with interferometric detection. However, the physical size of the source constrains how effectively radiation can be collected and focused for transmission through a small area. In principle, samples slightly smaller than the ca. $10 \mathrm{~mm}$ beam diameter on a commercial FTIR instrument employing a thermal source can be measured by using an aperture to physically restrict the beam size, but with a consequent sacrifice in signal intensity. Successful measurements on single protein crystals have employed infrared microscopes (see below) that can focus the beam from a commercial FTIR instrument further, to a few hundred $\mu \mathrm{m}$.

Although the majority of reported infrared measurements on protein crystals have used thermal sources, synchrotron radiation and lasers are potentially important alternatives. These nonthermal sources can typically be focused to smaller spot sizes, approaching the diffraction limit. This creates opportunities for measurements on crystals with dimensions on the order of the wavelength $(3-20 \mu \mathrm{m}$ in the mid-infrared region). Each source has special characteristics that must be taken into account.

A number of infrared beamlines are now available at synchrotron light sources and the lower panel of Fig. 2 displays a typical experimental signal. Note that the low frequency signal shown is instrumentally limited-the actual output continues to increase at low frequencies, allowing measurements into the $\mathrm{THz}$ region below $100 \mathrm{~cm}^{-1}$ with an appropriate detector. This contrasts with the blackbody distribution from a thermal source, which peaks near the temperature of the source and drops rapidly at lower frequencies. Other differences with respect to a benchtop thermal source include a higher power, and a significant degree of polarization evident in Fig. 2. The latter characteristic will be an important factor to take into account for quantitative polarization measurements on oriented crystals. Finally, it is important to note that fluctuations in source intensity are significantly larger than for a thermal source, and may be the limiting noise source.

Pulsed lasers are increasingly finding applications in infrared spectroscopy, including time-resolved pump-probe [21-25] and twodimensional infrared spectroscopy [26-28]. Infrared pulses are typically generated by difference frequency mixing of two short visible laser pulses in a nonlinear crystal. The spectral distribution is constrained by the temporal width of the pulse according to the uncertainty principle, so that the spectral distribution of pulses with temporal widths on the order of $100 \mathrm{fs}$ covers a few hundred $\mathrm{cm}^{-1}$. To our knowledge, pulsed laser infrared sources have not yet been applied to measurements on protein crystals, but they share with synchrotron light sources the capability for focusing approaching the diffraction limit, as well as a high degree of polarization and large intensity fluctuations. It is conceivable that tunable quantum cascade lasers operating in the mid-infrared will also become useful sources for spectroscopic measurements on protein crystals as the speed and range of frequency tuning continue to improve.

\subsection{Frequency selection}

The high signal-to-noise ratio needed to detect the small spectral differences associated with biological processes demands a strategy for simultaneously obtaining signal from many frequencies in parallel. FTIR instruments achieve this spectral multiplexing with a single-element detector through the use of a Michelson interferometer, which allows frequency selection without the signal attenuation that would result from measuring one frequency at a time [29]. Because of the difficulty of efficiently focusing light from a large area thermal source to a small area, dispersive instruments that rely on monochromators with narrow entrance and exit slits for high spectral resolution have much lower throughput are now rarely used for infrared spectroscopy. Commercial FTIR instruments represent a mature technology that forms the mainstay of biomolecular infrared spectroscopy, including published measurements on protein crystals.

In contrast, spectroscopic measurements with infrared lasers employ monochromators that select frequencies by using a grating to disperse the spectral energy distribution across the elements of a detector array $[21,23,25,30]$. In this case, the use of multiple elements enables simultaneous recording of signals at multiple frequencies. Normalization to the spectrum of the incident infrared pulse simultaneously recorded on a second reference monochromator, coupled with signal averaging over multiple pulses, effectively corrects for the large intensity fluctuations inherent to this source.

The optimal frequency selection method with a synchrotron infrared source is less apparent. The adoption of commercial FTIR instruments at existing beamlines is a practical choice that makes the full frequency spectrum of the source available to end users. However, fluctuations in source intensity preclude the attainment of signal-to-noise levels comparable to those that would be possible with a thermal source under equivalent conditions. Monochromator-based detection schemes analogous to those used with laser-based infrared systems would better compensate source noise fluctuations over a restricted frequency range, and might provide a more effective approach for biomolecular spectroscopy.

\section{Infrared microspectroscopy}

Although large numbers of crystals can be packed between IRtransparent windows to fill the large beam diameter of a standard FTIR sample chamber (ca. $10 \mathrm{~mm}$ ), measurements on single protein crystals have used infrared microscopes to focus IR beams to diameters approaching realistic crystal dimensions. Infrared microscopes have been available since the 1950s [31]. Early applications

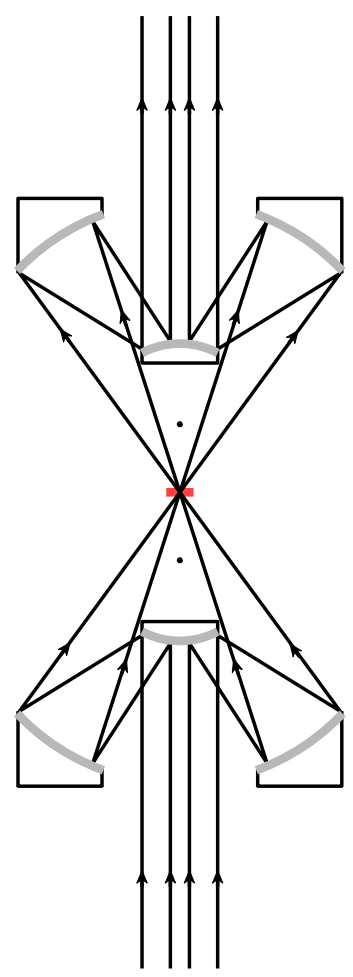

Fig. 3. Idealized ray trace through a pair of Schwarzschild objectives, with a protein crystal (size exaggerated) indicated in red at the common focal point, illustrates a typical arrangement for infrared transmission measurements. The common centers of curvature for the spherical reflective surfaces (gray) are indicated above and below the sample. An adjustable aperture in the image plane (not depicted) selects the measured sample area. The use of reflective optics ensures coincident image formation over a large frequency range. 
include Fraser's polarized IR measurements on oriented DNA fibers [32] (see Section 6.3) and Wegmann's identification of nucleic acid phosphate vibrations in mitochondria [33], well in advance of the enunciation of the endosymbiont hypothesis.

Although the optical design of microspectrophotometers for the visible and infrared regions is similar, one crucial difference is the need to form an image and maintain its position over a wide frequency range, so that an operator aligning the optical system "by eye" using visible light can be confident that the infrared image forms at the same position as the visible image. The practical means to meet this challenge is to use reflective optics as extensively as possible, thus ensuring optical throughput over a wide spectral range and minimizing chromatic aberration.

Commercial IR microscopes usually employ the Schwarzschild configuration (Fig. 3), which nests a convex reflecting surface within a concave surface to form a focusing optic equivalent to a lens [34]. The classical Schwarzschild objective uses concentric spherical surfaces, the ratio of whose radii is the square of the golden mean when the image forms at infinity [35]. In addition to eliminating chromatic aberration, this design eliminates spherical aberration in third order, allowing the use of a relatively large numerical aperture. Placement of an aperture stop at the center of curvature also eliminates coma, astigmatism, and distortion [36], but reduces the effective working distance and is not incorporated in commercial infrared microscopes. These advantages, and the simplicity of the design, more than compensate for partial obscuration by the support of the convex mirror, which reduces the fraction of beam energy in the central maximum of the diffraction spot [37].

An adjustable aperture in the image plane controls the area of the sample from which light is collected. Infrared light that passes slightly outside the visible image of the aperture at the sample position may also be collected because of diffraction or because of chromatic aberration introduced by refractive windows used to contain or support the sample. Some designs incorporate a second aperture before the sample to reduce diffraction effects. For protein crystals, additional aperturing in the sample plane may effectively take place in spectral regions where the transmission of the aqueous solution in which the crystal is typically immersed is lower than the transmission of the crystal itself.

The aperture and sample are finally imaged onto the detector element. Common infrared microscope designs use a $250 \times 250 \mu \mathrm{m}^{2}$ MCT detector element with unit magnification, so that this corresponds to the largest measurable sample area. The beam from a commercial FTIR spectrometer using a thermal source fills this area after focusing, and measurements on smaller areas require reducing the aperture area, with a proportionate sacrifice of experimental signal. In contrast, infrared beams available at synchrotron facilities can be focused more tightly, allowing measurement of much smaller samples. For example, the data in Fig. 4 were recorded on a photosystem II crystal with $30 \times 15 \mu \mathrm{m}^{2}$ area at the National Synchrotron Light Source (see Section 4). We anticipate that laser-based infrared sources will allow similar reductions in measurement area.

Commercial infrared microscopes allow convenient insertion of additional optical elements. Infrared polarizers consisting of arrays of parallel gold wires fabricated on a transparent substrate provide extinction ratios exceeding 100:1 and enable accurate measurements of infrared dichroism for oriented molecular ensembles such as single crystals. Optical filters may be used to restrict the spectral range, for example to remove residual leakage of the HeNe FTIR alignment laser for measurements on highly light-sensitive samples. On the other hand, commercial microscopes may require minor modifications to allow the introduction of visible lasers into the optical train for measurements of light-triggered spectral differences.

Because the Schwarzschild objective acts equivalently to a lens placed at the common center of curvature of the reflective surfaces, it has an intrinsically long working distance that facilitates insertion of sample control hardware such as flow cells (Fig. 6), cryostats, goniometers, and cryostream nozzles (Fig. 5). However, these

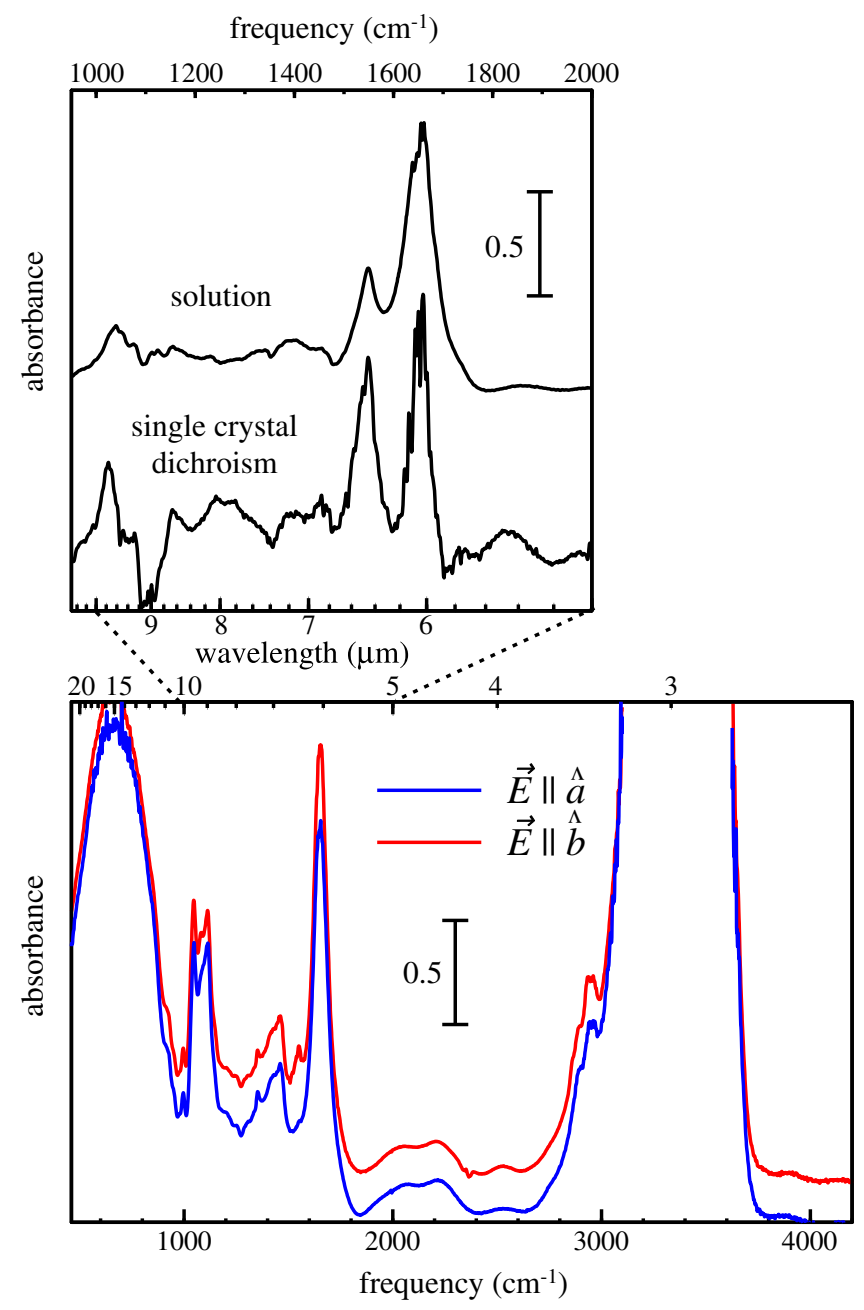

Fig. 4. The lower panel displays absolute absorption spectra recorded on the $\{001\}$ face of a single crystal of photosystem II (see [111] for experimental details) suspended in mother liquor between two calcium fluoride windows, with polarization parallel to the $a$ - and $b$-axes. Although unpolarized absorption by the mother liquor dominates the overall signal, polarized features in the absolute spectrum, such as the strongly $b$ polarized band visible at $1550 \mathrm{~cm}^{-1}$ reflect absorption by the protein. Subtraction of these two spectra clearly reveals dichroic signals corresponding to the amide I and amide II vibrations of the protein backbone, which are scaled up in the upper panel for comparison with the unpolarized signal measured on a solution. Crystal and solution data were recorded at ambient temperature at beamline U10A of the National Synchrotron Light Source.

additional capabilities may involve experimental compromises. For example, introduction of a cryostream into the beam path measurably increases fluctuations in optical signal, probably because of the higher refractive index of the cold gas, requiring careful adjustment to minimize turbulence. Many experiments require removing the collar that encloses the region of the optical path between objective and condenser on commercial instruments. However, instrument purge to remove absorption by $\mathrm{CO}_{2}$ and water vapor is compromised when the beam path between the Schwarzschild elements remains open to atmosphere during measurement.

\section{Single crystal measurement strategies}

\subsection{Transmission}

Infrared spectroscopy commonly relies on measuring light transmission through a sample, with the absorbance

$\mathcal{A}=-\log I / I_{0}$ 


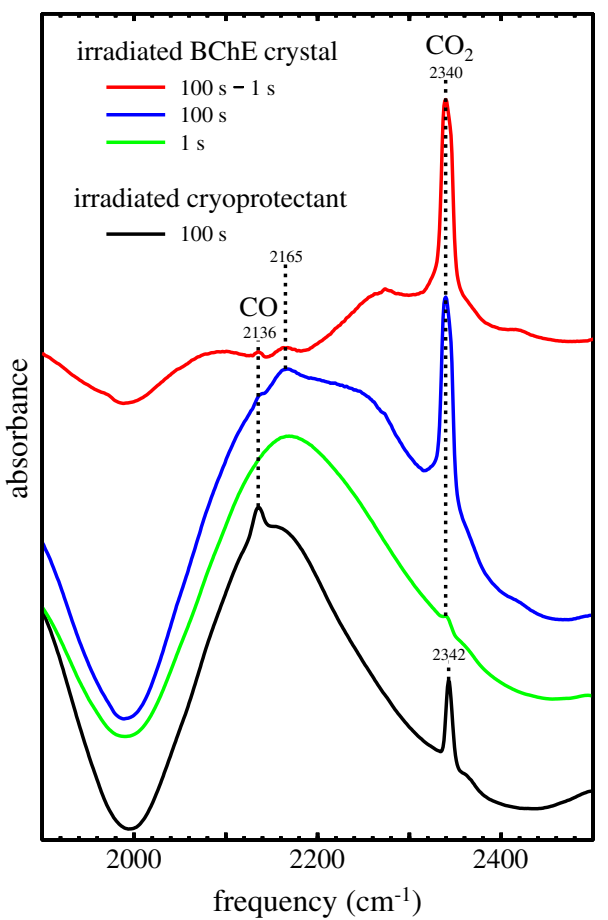

Fig. 5. Infrared absorption bands in irradiated single crystals of human butyrylcholinesterase $(\mathrm{BChE})$ increase with dose and reveal molecular species resulting from radiation exposure (see [113] for experimental details). The absorbed dose was $5.5 \mathrm{MGy}$ and $55 \mathrm{kGy}$ for the BChE crystals irradiated for $100 \mathrm{~s}$ (blue) and $1 \mathrm{~s}$ (green), respectively, as calculated with the program RADDOSE [114]. Subtraction of spectra recorded at high and low dose (red) minimizes solvent absorption and emphasizes spectral changes resulting from X-ray exposure. The prominent band appearing near $2340 \mathrm{~cm}^{-1}$ is consistent with the production of $\mathrm{CO}_{2}$. Weaker absorption near $2136 \mathrm{~cm}^{-1}$ may reflect a small population of $\mathrm{CO}$. A control measurement on the irradiated cryoprotectant solution reveals the same species after $100 \mathrm{~s}$ exposure (black), but with altered relative populations.

determined experimentally from the ratio of transmitted and incident irradiances, $I$ and $I_{0}$, respectively. The intensity

$I=I_{0} e^{-d / \delta}$

decreases exponentially with sample thickness $d$, with the absorption cross sections $\sigma_{j}(\bar{v})$ and concentrations $c_{j}$ of all molecules in the sample determining the penetration depth $\delta=1 / \sum_{j} \sigma_{j}(\bar{\nu}) c_{j} \ln 10$ and the absorbance

$\mathcal{A}=\sum_{j} \sigma_{j}(\bar{v}) c_{j} d \ln 10$

For oriented samples including crystals, it is crucial to keep in mind that the cross sections vary with the polarization as well as with the frequency of the incident radiation (Section 5).

Note that Eq. (3) omits scattering contributions, which vary as the fourth power of wavelength and are typically negligible in the midinfrared, even for samples that appear turbid in visible light. For example, infrared absorption measurements can often be performed at cryogenic temperatures without adding glass forming cosolvents such as glycerol that are usually required for absorption measurements with visible wavelengths.

As outlined above, infrared transmission measurements on protein crystals must satisfy two competing constraints. On one hand, water absorption (Fig. 1) limits practical infrared measurements below $1700 \mathrm{~cm}^{-1}$ on aqueous solutions to pathlengths shorter than 5$10 \mu \mathrm{m}$. On the other hand, radiation from a thermal infrared source cannot easily be focused onto an area smaller than a few hundred $\mu \mathrm{m}$, even using an infrared microscope (Fig. 3).
For this reason, high-quality infrared transmission measurements below $1800 \mathrm{~cm}^{-1}$ have been demonstrated only for crystals with very high aspect ratios, but such favorable cases are relatively rare [38-40]. A more common approach has been to arrange large numbers of small crystals to fill a large beam area[41-46]. These measurements have focused on spectroscopic comparison of crystals with solutions and did not exploit the molecular orientation through polarized IR measurements.

The ability to focus radiation from a nonthermal source close to the diffraction limit is an important opportunity that has been littleexploited. Polarized infrared spectra of a $15 \times 30 \mu \mathrm{m}^{2}$ photosystem II crystal recorded at an infrared beamline (Fig. 4) demonstrate the possibility. Absorption by the mother liquor in which the crystal is suspended dominates the absolute spectrum in Fig. 4, in part because the distance between sample cell windows is larger than the crystal thickness. In addition to strong water absorption near 700, 1640, and $3400 \mathrm{~cm}^{-1}$, as noted above (Fig. 1), polyethylene glycol, used to initiate protein crystallization, also absorbs strongly near $1100 \mathrm{~cm}^{-1}$. However, features attributable to protein absorption can be distinguished on the basis of their strong polarization. For example, the characteristic amide II band of the polypeptide backbone is visible as a strongly $b$-polarized feature at $1549 \mathrm{~cm}^{-1}$, on the wing of the $1640 \mathrm{~cm}^{-1}$ band primarily due to $\mathrm{HOH}$ bending.

These restrictions are relaxed in a spectral "window" from 1800 to $2800 \mathrm{~cm}^{-1}$, between the water stretching and bending vibrations, where solvent absorption is relatively low (Fig. 1), allowing measurements on crystals up to about $100 \mu \mathrm{m}$ in thickness. Fig. 5 shows absorption spectra recorded in this region on single crystals of human butyrylcholinesterase (BChE) flash-cooled in cryoloops and measured at $100 \mathrm{~K}$. Cryosolvent absorption dominates the spectra, which exhibit a broad water absorption band near $2150 \mathrm{~cm}^{-1}$. However, an additional sharp, asymmetric band appears at $2340 \mathrm{~cm}^{-1}$ following irradiation with $13.2 \mathrm{keV}$ photons at a crystallography beamline (see Fig. 5 caption for further experimental details), along with weaker features. Decarboxylation of acidic amino acid residues is one common mechanism for radiation damage during prolonged X-ray exposure [47], and the $2340 \mathrm{~cm}^{-1}$ frequency is consistent with the asymmetric stretching vibration of the $\mathrm{CO}_{2}$ thus generated.

Polypeptide vibrations contribute little signal in this region, with the no table exception of the cysteine $\mathrm{S}-\mathrm{H}$ bond, which typically vibrates in the $2600-2700 \mathrm{~cm}^{-1}$ range [22,48-50]. Both frequency and cross section are highly sensitive to hydrogen bonding [48,51]. IR measurements on hemoglobin crystals demonstrated the capability to distinguish contributions from individual cysteine residues based on differing polarization [52]. C-D vibrational frequencies also lie within the water window and are emerging as flexible probes of targeted protein sites, facilitated by advances in molecular biology that enable specific deuteration of selected amino acid residues [53,54].

The water absorption window has been exploited most heavily to probe protein ligands. Small molecules including $\mathrm{CO}, \mathrm{NO}, \mathrm{N}_{3}^{-}$, and $\mathrm{CN}^{-}$ are particularly valuable probes for metal centers [55,56]. Their stretching vibrations occur in the $1800-2300 \mathrm{~cm}^{-1}$ range, because of the high bond order, and exhibit strong absorption, sometimes enhanced by coordination to the metal sites. $\mathrm{CO}_{2}$ and $\mathrm{N}_{2} \mathrm{O}$ are important metabolites whose antisymmetric stretching frequencies absorb in the water window $[57,58]$.

\subsection{Difference measurements}

The intrinsically high sensitivity of modern infrared techniques is a crucial advantage for applications to biomolecular systems, including protein crystals. In favorable cases, careful spectral subtraction can detect structural changes at the level of individual bonds, even within macromolecules containing thousands of atoms suspended in a highly absorbing solvent $[59,60]$. More routinely, solvent subtraction is nearly always required to reveal protein contributions to the infrared signal. 
Polarized spectra of photosystem II crystals offer a simple illustration. As noted above, solvent absorption dominates the absolute spectra in the lower panel of Fig. 4. However, subtraction of spectra recorded with light polarized parallel to the $a$ - and $b$-axes eliminates the unpolarized solvent contribution, revealing the characteristic amide I and amide II vibrations of the polypeptide backbone (upper panel). The amide I vibration at $1660 \mathrm{~cm}^{-1}$, in particular, is completely obscured by the solvent band near $1640 \mathrm{~cm}^{-1}$ in the absolute spectrum, although the amide II band is visible on its shoulder in the $b$-polarized spectrum. The spectrum of a (randomly oriented) photosystem II solution, included for comparison in the upper panel, was also determined by subtraction of a separately recorded spectrum of the mother liquor. In this case, the relative weights of the spectra were adjusted by visual inspection to generate a flat background, while the crystal polarization subtraction was unweighted.

The frequencies and band shapes are similar, as shown. However, the relative intensities differ, presumably reflecting the known difference in orientation of the transition dipoles for the amide I and II vibrations [61]. We emphasize that hundreds of unresolved vibrational resonances contribute to each of these bands, since each peptide bond contributes equivalently to the observed signals. Thus, although these data demonstrate that nonthermal sources can be used to obtain infrared data on crystals with sizes approaching the diffraction limit, the signal-to-noise level achieved is not yet adequate to detect structural changes at the level of individual bonds. More detailed investigations will require compensating for fluctuations in source intensity, which are significantly larger than those observed using a thermal source.

Similarly, subtraction of spectra recorded on two BChE crystals with differing radiation doses (Fig. 5) reduces the contribution of the broad solvent band near $2150 \mathrm{~cm}^{-1}$ and helps to expose the contributions of $\mathrm{X}$-ray-generated molecular species responsible for relatively sharp bands at 2136, 2165, and $2340 \mathrm{~cm}^{-1}$. Some uncertainty surrounds broader features, because variations in the lineshape of the solvent feature in the two crystals may result in baseline fluctuations after subtraction. Polarization difference spectra on photosystem II crystals (Fig. 4) also exhibit baseline artifacts, in this case due to fluctuations in the source spectrum. Moreover, the noise level is not adequate for detecting changes at the level of individual bonds, on the order of $1 \%$ of the total protein absorbance. Direct difference measurements on the same crystal using a stable source reduce baseline uncertainties and allow sensitivity to much smaller changes.

Small molecules can diffuse through the solvent channels of a protein crystal, and crystallographic studies often exploit this property for structural determination of reaction products. Similarly, placement of a

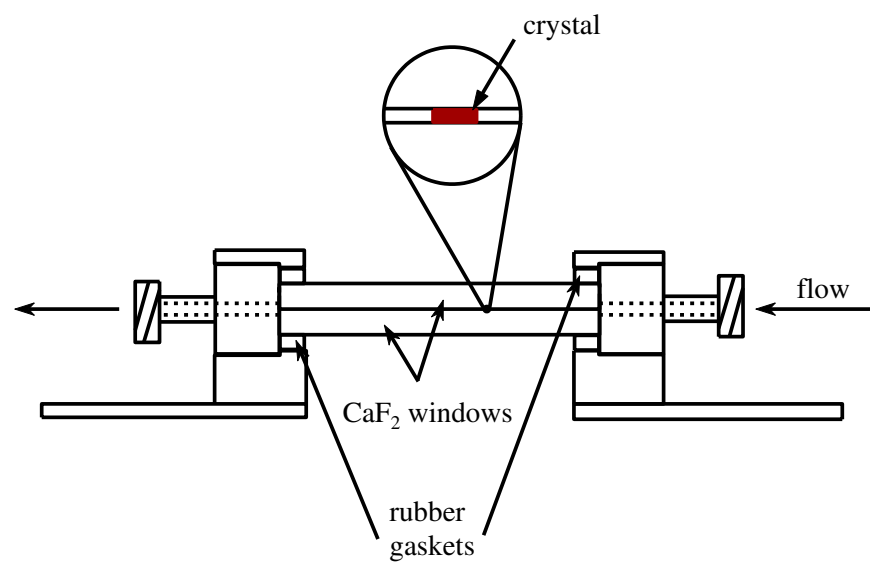

Fig. 6. Assembly of a flow cell designed for infrared measurements on protein crystals sandwiches crystals and mother liquor between two infrared-transparent windows. Channels in the cell body allow exchange of the solution bathing the crystal, enabling measurement of spectral changes in response to dissolved ligands. protein crystal in a flow cell with windows that are transparent in the infrared allows the calculation of spectral differences before and after introduction of solvent containing reactive ligands. The flow cell depicted in Fig. 6 was used to determine infrared spectral changes upon reaction with small molecules including $\mathrm{CO}, \mathrm{CN}^{-}$, and $\mathrm{N}_{3}^{-}$[52,62-64]. We also demonstrated the ability to reversibly titrate crystal $\mathrm{pH}$ over a wide range [63]. An additional advantage of this cell is that it can be assembled with a small wedge between the windows so that the application of a gentle flow moves the crystal until it lodges between the windows, minimizing the contribution of solvent absorbance and ensuring that it remains stationary, improving the quality of the difference measurements.

Fig. 7 shows the results of a pH titration on a single crystal of MbCO. Subtraction reveals not only the $\mathrm{C}-\mathrm{O}$ fundamental, but also the first overtone, whose absorbance is two orders of magnitude weaker. To our knowledge, the direct $0 \rightarrow 2$ overtone transition has not been directly observed for a protein in solution, but the observed anharmonic shift is consistent with the frequency difference between $0 \rightarrow 1$ and $1 \rightarrow 2$ fundamental transitions observed using nonlinear IR techniques [65]. The high ratio of protein to solvent signal in the crystalline phase undoubtedly facilitates successful observation of the overtone transition. The protein concentration calculated from the lattice constants reported [66] for the $P 2_{1}$ crystal form of the MbCO crystal shown here is $49 \mathrm{mM}$.

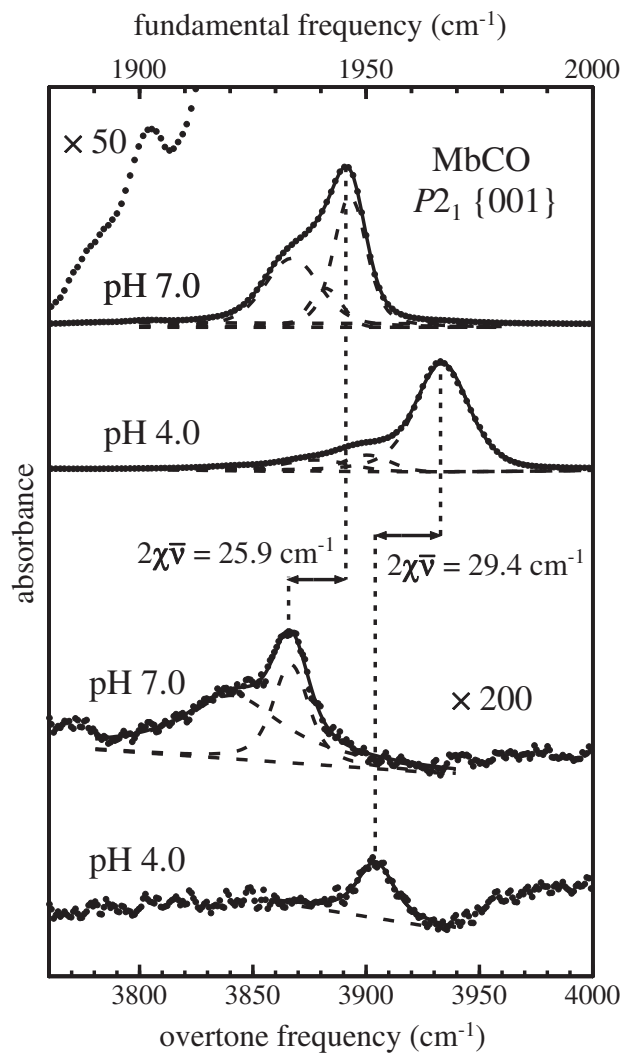

Fig. 7. Infrared spectra of myoglobin exhibit multiple features associated with the stretching of CO bound to the heme iron. Spectra were recorded at ambient temperature on the face $\{001\}$ of a single crystal mounted in the flow cell depicted in Fig. 6. In addition to the ${ }^{12} \mathrm{C}-\mathrm{O}$ fundamental, whose peak frequency shifts from $1945 \mathrm{~cm}^{-1}$ to $1966 \mathrm{~cm}^{-1}$ when the $\mathrm{pH}$ of the solution bathing the crystal is reduced from 7 to 4 , the data reveal a weak feature at $1903 \mathrm{~cm}^{-1}$, associated with the ${ }^{13} \mathrm{C}-\mathrm{O}$ fundamental, as well as higher frequency features two orders of magnitude weaker that we attribute to the first overtone of the ${ }^{12} \mathrm{C}-\mathrm{O}$ stretch. All data are dichroic signals resulting from a double subtraction: first, spectral differences were recorded before and after exchanging the mother liquor with a solution containing $\mathrm{CO}$ and sodium dithionite, then the resulting spectrum recorded with polarization parallel to the $b$-axis is subtracted from the analogous $a$-polarized spectrum. The polarization difference removes uncorrected water vapor peaks to reveal the $\mathrm{C}-\mathrm{O}$ overtones. Spectra displayed in Figs. 7, 8, and 10 were recorded on crystals of sperm whale myoglobin grown in the $P 2{ }_{1}$ space group from ammonium sulfate solutions. 
The results in Fig. 7 underline the extremely high infrared spectral sensitivity achievable using a stable light source focused to the area of the crystal. We anticipate that suitable matching of crystals and light sources will allow recording of high quality difference spectra in response to perturbations such as photoreactions or electrochemical titration $[59,60]$.

\subsection{Reflectance}

Transmission measurements on aqueous solutions of biological molecules in the $3000-3700 \mathrm{~cm}^{-1}$ region are relatively rare. The strong absorbance from solvent $\mathrm{O}-\mathrm{H}$ stretching vibrations requires very short pathlengths $(\leq 1 \mu \mathrm{m})$ which are difficult to control accurately. Investigations in this region have used transmission measurements on partially hydrated protein films to identify spectral changes associated with individual water molecules involved in proton transfer chains $[22,67]$.

Reflectance measurements provide an alternative method to access strongly absorbing regions. Both real and imaginary components of the refractive index contribute to the reflectivity, but measurements over a wide frequency range enable the performance of an integral transform [29] that reveals the imaginary part of the index, which is proportional to the absorbance. Only a surface layer with a thickness on the order of the wavelength contributes to the signal.

Fig. 8 compares the reflectance spectrum recorded on a large single crystal of oxidized myoglobin (metMb), flash-cooled on a cryoloop without cryoprotectant in order to minimize solvent contributions to the reflectance, with spectra recorded on films of a concentrated solution of metMb and of ice, also frozen on cryoloops. The crystal spectrum clearly displays components that are not due to solvent. Frequencies near $2900 \mathrm{~cm}^{-1}$ are typical for $\mathrm{C}-\mathrm{H}$ stretching vibrations of alkanes and presumably represent contributions from amino acid side chains. These features are not evident in a "concentrated" metMb solution, and their prominent contribution to the crystal data reflects both the high protein concentration and the reduced solvent content in the crystalline environment. $\mathrm{O}-\mathrm{H}$ and $\mathrm{N}-\mathrm{H}$ vibrations of the protein

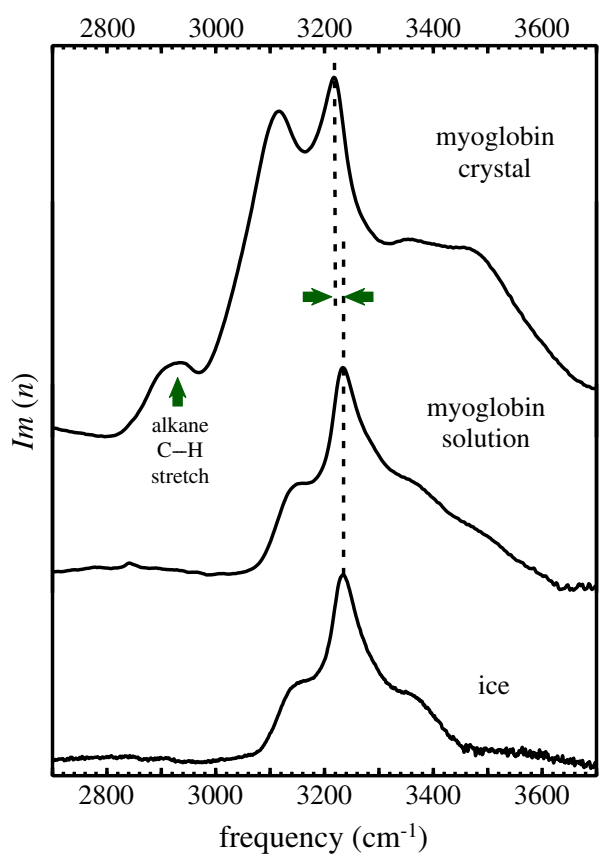

Fig. 8. Imaginary part of the refractive index determined from the reflectance of a single crystal of oxidized myoglobin (upper trace), and of frozen thin films of concentrated solutions of oxidized myoglobin and of ice (middle and lower traces). All samples were suspended on a cryoloop in an infrared microscope, with temperature stabilized by a $100 \mathrm{~K}$ nitrogen gas stream. The spectral intensity reflected from a gold mirror was used as a reference for each sample. and the ammonium sulfate in the mother liquor may account for additional differences between solvent and crystal spectra at higher frequencies. The peak of the ice spectrum appears $18 \mathrm{~cm}^{-1}$ lower in the crystal spectrum, which suggests slightly enhanced hydrogen bonding of crystal water in comparison with the bulk solvent.

\section{Quantitative analysis of polarized absorption measurements}

The absorption of polarized radiation probes the orientation of molecules or groups of atoms within molecules because the probability for inducing a molecular transition depends on the projection of the polarization direction ê of the incident radiation onto a transition dipole $e \vec{R}_{\text {if }}$ connecting initial and final states of the molecule. Specifically, in the long wavelength approximation, the individual resonances contributing to the molecular absorption cross section

$\sigma(\bar{v})=4 \pi^{2} \alpha \sum_{i f} p_{\mathrm{i}}\left(\hat{e} \cdot \vec{R}_{i f}\right)^{2} \bar{v} \mathcal{L}\left(\bar{v}-\bar{v}_{i f}\right)$

are centered at frequencies determined by the energy separation $h c \bar{\nu}_{i f}=E_{f}-E_{i}$ between initial and final molecular states. The line shape function in Eq. (4) has unit area $\left(\int \mathcal{L}(\bar{\nu}) d \bar{\nu}=1\right)$ and $\alpha=e^{2} / 4 \pi \varepsilon_{0} \hbar c$ is the fine structure constant.

In the harmonic approximation, the initial and final vibrational states $\left|\chi_{\mathrm{i}}\right\rangle$ and $\left|\chi_{\mathrm{f}}\right\rangle$ can be described as products of harmonic oscillator wave functions and the transition dipole

$e \vec{R}_{i f}=\left\langle\chi_{\mathrm{f}}|e \vec{R}| \chi_{\mathrm{i}}\right\rangle=\left(\frac{\hbar}{2 \omega_{\alpha}}\right)^{1 / 2} \frac{\partial \vec{R}}{\partial Q_{\alpha}}\left(n_{\alpha}+1\right)+\ldots$

for fundamental excitations $n_{\alpha} \rightarrow n_{\alpha}+1$ of a single normal mode within the same electronic manifold is determined to first order by derivatives of the molecular dipole $e \vec{R}$ with respect to mass-weighted normal coordinates $Q_{\alpha}$. Substitution of $n_{\alpha}$ for $n_{\alpha}+1$ in Eq. (5) gives the transition dipole for $n_{\alpha} \rightarrow n_{\alpha}-1$ and all other transition dipoles vanish to first order in the harmonic approximation.

The total cross section resulting from substituting transition dipoles corresponding to both absorption and stimulated emission into Eq. (4) has a temperature independent form

$\sigma(\bar{v})=\frac{\alpha h}{2 c} \sum_{\alpha}\left|\hat{e} \cdot \frac{\partial \vec{R}}{\partial Q_{\alpha}}\right|^{2} \mathcal{L}\left(\bar{v}-\bar{v}_{\alpha}\right)$

with a single resonance centered at the frequency of each normal mode. The absorbance

$$
\begin{aligned}
\mathcal{A}_{\text {iso }}(\bar{v}) & =\sum_{j} c_{j} d \ln 10 \sum_{\alpha} \frac{\alpha h}{6 c}\left|\frac{\partial \vec{R}_{j}}{\partial Q_{\alpha}}\right|^{2} \mathcal{L}\left(\bar{v}-\bar{v}_{\alpha}\right) \\
& =\sum_{j \alpha} \epsilon_{j \alpha}(\bar{v}) c_{j} d
\end{aligned}
$$

measured on a randomly oriented ensemble of molecules averages uniformly over all molecular orientations and the isotropic extinction coefficient $\epsilon_{j \alpha}(\bar{\nu})$ depends only on the magnitude of the vibrational transition dipole. More generally, we can define a unit vector $\hat{\mu}_{j \alpha}$ parallel to the transition dipole, and the measured absorbance

$\mathcal{A}(\bar{v})=3 \sum_{j \alpha} \epsilon_{j \alpha}(\bar{v}) c_{j} d\left\langle\left(\hat{e} \cdot \hat{\mu}_{j \alpha}\right)^{2}\right\rangle$

is a population-weighted average of the isotropic absorbance $\epsilon_{j \alpha}(\bar{\nu}) c_{j} d$ over the angular distribution of $\hat{\mu}_{j \alpha}$ relative to the polarization direction $\hat{e}$ 


\subsection{Absorption in anisotropic media}

Accurate quantitative interpretation of polarized absorption measurements requires consideration of the effect of the anisotropic environment on the propagation of electromagnetic radiation $[68,69]$. In particular, the naive expectation that maximal absorption occurs when the polarization of the incident light aligns with the transition dipole is not correct in general.

In an isotropic medium, plane waves of any polarization state satisfy Maxwell's Equations. In contrast, an anisotropic medium supports propagation of only two independent polarizations $\hat{\mathrm{e}}_{1}(\bar{\nu})$ and $\hat{e}_{2}(\bar{\nu})$ with distinct refractive indices $n_{1}(\bar{v})$ and $n_{2}(\bar{\nu})$. In a uniaxial crystal, one polarization is always perpendicular to the optic axis and the corresponding wave is called the ordinary wave, while the other wave solution to Maxwell's Equations has an orthogonal polarization and is called the extraordinary wave. Linear combinations of these two modes produce waves whose electric field direction varies as it propagates because the relative phase of the two components changes with distance.

Eq. (9) relates the absorbances $\mathcal{A}_{1}$ and $\mathcal{A}_{2}$ of these two modes to the orientations $\hat{\mu}_{j}$ of the transition dipoles relative to the mode polarizations $\hat{e}_{1}$ and $\hat{e}_{2}$. The intensity of each wave decreases exponentially according to Eq. (1), and the measured absorbance [70]

$\mathcal{A}=-\log \frac{I}{I_{0}}=-\log \left[f_{1} 10^{-\mathcal{A}_{1}}+f_{2} 10^{-\mathcal{A}_{2}}\right]$

of light with incident intensity $I_{0}$ depends on these absorbances and on the fractions $f_{1}$ and $f_{2}$ of the incident energy that couple to the two allowed modes and are subsequently transmitted through the anisotropic medium. In general, there is some reflection at the front and rear faces of the crystal, so that $f_{1}+f_{2}<1$.

A typical experimental situation involves normally incident light whose polarization plane lies an angle $\chi$ from the polarization $\hat{e}_{1}$ of one of the allowed radiation modes. Given an incident intensity $I_{0}$, this light will induce transmitted beams of polarization $\hat{e}_{1}$ and $\hat{e}_{2}$ with intensities proportional to $I_{0} \cos ^{2} \chi$ and $I_{0} \sin ^{2} \chi$, respectively, that are attenuated at a rate determined by the absorbances $\mathcal{A}_{1}$ and $\mathcal{A}_{2}$ in Eq. (10). The total transmitted intensity $I$ determines the measured absorbance

$A(\chi)=-\log \left[\mathcal{T}_{1} 10^{-\mathcal{A}_{1}} \cos ^{2} \chi+\mathcal{T}_{2} 10^{-\mathcal{A}_{2}} \sin ^{2} \chi\right]$.

Practical measurements are usually conducted with radiation incident normal to the surface, where basic optics predicts that transmission coefficients are least sensitive to polarization [71]. In this case, it is usually a reasonable practical assumption to take $\mathcal{T}_{1} \approx \mathcal{T}_{2}$, neglecting the small differences that must exist even at normal incidence because of the differing indices of refraction. (If needed, the measured absorbance $\mathcal{A}_{1}^{*}=\mathcal{A}_{1}-\log \mathcal{T}_{1}$ can be corrected for this small effect.) Similarly, the difference in refractive index is usually small enough to justify neglecting small deviations between the direction of ordinary and extraordinary waves [72], and can usually be eliminated by measuring absorption along a low-index crystal direction in crystal symmetries higher than triclinic. Highly sensitive measurements are required to detect the circular dichroism of biological macromolecules in the infrared [73], suggesting that this potential complicating factor can also be safely neglected in comparison with the much larger linear dichroism present in crystalline samples.

Measurements of the antisymmetric stretching frequency of azide $\left(\mathrm{N}_{3}^{-}\right)$bound to a $P 2_{1}$ myoglobin crystal as a function of polarizer orientation (Fig. 9) compare well with Eq. (11). Note that Eq. (11) predicts, for all transitions, that maximum and minimum absorbances are observed at $\chi=0^{\circ}$ and $\chi=90^{\circ}$, when the incident polarization aligns with the polarizations of the allowed radiation modes. Symmetry considerations often dictate that these directions correspond with crystallographic axes. However, these directions usually

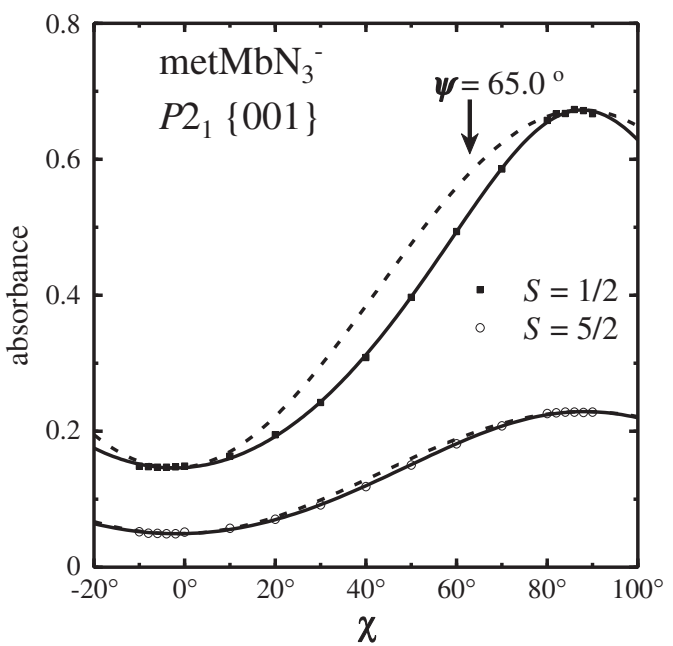

Fig. 9. The peak absorbance of antisymmetric $\mathrm{N}_{3}^{-}$stretching vibrations bound to the heme group in $P 2_{1}$ myoglobin crystals follow the angular dependence predicted by Eq. (11) (solid line). Deviation from the cosine-squared dependence predicted in the low absorbance limit of Eq. (12) (dashed line) becomes particularly noticeable for the dominant feature due to $\mathrm{N}_{3}^{-}$bound to low spin heme. The arrow indicates the projected orientation of the transition dipole determined from the maximum and minimum absorbances [62] according to Eqs. (18) and (21). Peak absorbance values result from curve fitting of spectra recorded at each polarizer orientation. Fig. 10 shows the spectra recorded at $\chi=-2^{\circ}$ and $\chi=88^{\circ}$.

do not correspond with the orientations of individual vibrational dipoles, which lie at a variety of orientations for complex molecules. For example, maximal alignment between the incident polarization and the transition dipole occurs at the angle marked by the vertical arrow in Fig. 9.

When both absorbances are small $\left(\mathcal{A}_{1} \ll 1, \mathcal{A}_{2} \ll 1\right)$, the absorbance

$A(\chi)=\mathcal{A}_{1} \cos ^{2} \chi+\mathcal{A}_{2} \sin ^{2} \chi$.

displays a simpler sinusoidal dependence on polarization direction. Fig. 9 demonstrates that infrared absorption measurements on protein crystals do not correspond to this limit in general, and illustrates the strong deviation from sinusoidal behavior observed for larger absorbances. A more subtle consequence of the nonlinear dependence on $\mathcal{A}_{1}$ and $\mathcal{A}_{2}$ in Eq. (11) is that the infrared spectrum recorded on a strongly absorbing crystal at an arbitrary orientation and polarization is not the simple interpolation between the absorbance of the two electromagnetic modes predicted in the low absorbance limit of Eq. (12). Unpolarized spectra will display significant lineshape distortions that increase with crystal thickness.

Proper understanding of the behavior of electromagnetic radiation in the crystal, although possibly counterintuitive, guides and simplifies experimental measurements on oriented crystals. Crystal (or polarizer) orientation can be optimized simply by searching for absorbance extrema as a function of polarizer orientation. Once the orientation is established, subsequent measurements need only be conducted at $\chi=0^{\circ}$ and $\chi=90^{\circ}$. These measurements are sufficient to determine the two experimentally accessible absorption spectra $\mathcal{A}_{1}(\bar{v})$ and $\mathcal{A}_{2}(\bar{v})$.

The differing absorption of the two polarization states probes the orientations of vibrational transition dipoles and provides useful information about molecular structure and orientation. The precise information available will depend on the crystal symmetry. In an ideal crystalline environment, the average

$\left\langle(\hat{e} \cdot \hat{\mu})^{2}\right\rangle=\frac{1}{m N} \sum_{j=1}^{m} \sum_{k=1}^{N}\left(\hat{\mu}_{\alpha j k} \cdot \hat{e}\right)^{2}$

in Eq. (9) extends over a discrete number of molecular orientations. Symmetry operators in the crystal space group generate $N$ copies of 
each molecular species appearing in the asymmetric unit of the crystal. Eq. (13) also includes $m$ sites in the asymmetric unit that are spectroscopically indistinguishable, that is, $\epsilon_{\alpha}(\bar{\nu})$ is identical for all $m$ sites, but they are not related by any crystallographic symmetry (it is not uncommon for multiple molecules to appear in the asymmetric unit of macromolecular crystals). The quantity $(\hat{\mu} \cdot \hat{e})^{2}$ is invariant under translation or inversion, and we can determine the form of the average in Eq. (13) by considering how $\hat{\mu}$ transforms under the $n$-fold rotations that generate the space group, as described in detail in the Appendix.

\subsubsection{Biaxial crystals}

The absence of symmetry axes in triclinic crystals has two consequences. First, the sum over $k$ in Eq. (13) contains a single term and the absorbance

$\mathcal{A}=\sum_{\alpha} 3 \epsilon_{\alpha} c d \frac{1}{m} \sum_{j=1}^{m}\left(\hat{\mu}_{\alpha j} \cdot \hat{e}\right)^{2}$

of light polarized along $\hat{e}$ depends only on the orientations of transition dipoles associated with the molecules in the asymmetric unit. A less fortunate consequence of the low symmetry is that the optic axes do not have a fixed orientation with respect to the crystallographic axes and must be determined experimentally (in principle at each frequency) by variable polarization measurements.

This potential experimental complication is alleviated for monoclinic or orthorhombic space groups, whose highest symmetry generators involve 2-fold rotations. For light incident normal to a symmetry axis, the allowed polarizations are parallel and perpendicular to the axis. In this situation, a coordinate system defined by the experimentally determined polarization directions $\hat{e}_{1}$ and $\hat{e}_{2}$ and the propagation direction $\hat{k}$ (Fig. 11) yields a convenient description of transition dipole orientations with respect to the crystallographic axes. Moreover, the quantity $(\hat{\mu} \cdot \hat{e})^{2}$ is invariant under twofold rotation for the allowed polarizations, so that Eq. (14) still holds. Thus, a representative transition dipole orientation $\hat{\mu}_{\alpha j}$ for each vibrational mode of each molecule in the asymmetric unit determines the polarized absorption properties.

A polar angle $\delta_{\alpha j}$, measured from $\hat{k}$, and an azimuthal angle $\psi_{\alpha j}$, measured from $\hat{e}_{1}$ in the plane normal to $\hat{k}$, specify the orientation of the transition dipole

$\hat{\mu}_{\alpha j}=\left(\sin \delta_{\alpha j} \cos \psi_{\alpha j}, \sin \delta_{\alpha j} \sin \psi_{\alpha j}, \cos \delta_{\alpha j}\right)$

in this coordinate system (Fig. 9). Absorption measurements on an oriented single crystal provide quantitative information on the transition dipole orientation. The absorption spectrum

$\overline{\mathcal{A}}_{\mathrm{avg}}=\frac{1}{2}\left(\mathcal{A}_{1}+\mathcal{A}_{2}\right)=\sum_{\alpha} \frac{3}{2} \epsilon_{\alpha} c d \frac{1}{m} \sum_{j=1}^{m} \sin ^{2} \delta_{\alpha j}$

determined by averaging the spectra recorded with the two allowed polarizations depends on the angles of the transition dipole with respect to the propagation direction. Specifically, a spectrally resolved feature corresponding to mode $\alpha$ in the unpolarized crystal absorbance differs by an orientation factor

$R_{\alpha}=\frac{3}{2} \frac{1}{m} \sum_{j=1}^{m} \sin ^{2} \delta_{\alpha j}$

from the absorbance $\mathcal{A}=\epsilon_{\alpha} c d$ of a randomly oriented molecular ensemble with the same concentration and pathlength.

In this context, we emphasize that orientation effects will always influence absorption measurements on oriented single crystals, even without controlling the polarization of the incident light. As an obvious example, a transition dipole oriented parallel to the propagation direction $\hat{k}\left(\delta_{\alpha j}=0^{\circ}\right)$ makes no contribution to the observed absorption spectrum. In the low absorbance limit, measurements using randomly polarized light will yield the polarizationaveraged spectrum (Eq. (16)). As a result, the relative intensities of spectral features may vary by a factor (Eq. (17)) ranging from 0 to 1.5 due to orientation and caution should be exercised before attributing intensity differences observed between crystal and solution spectra as indicators of structural differences. A similar caveat applies to Raman spectra of oriented single crystals [74]. Further differences may appear for strongly absorbing crystals, for which the predicted absorbance, obtained by averaging the argument of the logarithm in Eq. (11) over $\chi$, will differ from the absorbance (Eq. (16)) obtained from averaging the expression (Eq. (12)) for the low absorbance limit. The dichroic index

$\Pi=\frac{\mathcal{A}_{1}-\mathcal{A}_{2}}{\mathcal{A}_{1}+\mathcal{A}_{2}}$

is more straightforward to interpret than $R$, because it does not require independent experimental determinations of the crystal thickness $d$ or the isotropic extinction coefficient $\epsilon_{\alpha}$. Explicit substitution of the transition dipole orientation shows that the dichroic index

$\Pi=\frac{\sum_{j} \sin ^{2} \delta_{\alpha j} \cos 2 \psi_{\alpha j}}{\sum_{j} \sin ^{2} \delta_{\alpha j}}$

depends on the azimuthal angle $\psi_{\alpha j}$ through the averaged value of cos $2 \psi_{\alpha j}$, weighted by the relative contribution of each transition dipole in the asymmetric unit to the average absorbance.

Experimental determinations of $R$ and $\Pi$ strongly constrain the possible orientations of the transition dipole. This is seen most clearly for a single molecule in the asymmetric unit $(m=1)$, when measurements of

$R_{\alpha}=\sin ^{2} \delta_{\alpha}$

and

$\Pi_{\alpha}=\cos 2 \psi_{\alpha}$

directly specify allowed transition dipole orientations $\left(\delta_{\alpha}, \psi_{\alpha}\right)$ in the crystallographic coordinate system. In the absence of other information, there is a remaining ambiguity in the dipole orientation because there are four values $\pm \psi_{\alpha}, \pm\left(\pi-\psi_{\alpha}\right)$ of the azimuthal and two values $\delta_{\alpha}, \pi-\delta_{\alpha}$ of the polar angle that satisfy the experimental constraints. Stereochemical information or, ideally, polarization measurements on different crystal forms can further constrain possible transition dipole orientations.

Accurate quantification of the polarization properties $R_{\alpha}$ and $\Pi_{\alpha}$ associated with an individual vibration may require additional data analysis such as curve fitting. Uncertainties may result from calculation of the dichroic index according to Eq. (18) at each frequency, because of unresolved spectral contributions. For example, antisymmetric vibrations of $\mathrm{N}_{3}^{-}$bound to high and low-spin hemes in Fig. 10 are spectrally resolved from each other. The calculated dichroic index is nearly constant across the profile of the dominant low-spin peak at $2024 \mathrm{~cm}^{-1}$, but varies significantly at higher frequencies. Coupled with subtle polarization differences visible in the lineshape of the high-spin peak at $2045 \mathrm{~cm}^{-1}$, this suggests unresolved spectral contributions with differing polarization properties. For example, a small percentage of free azide in the solvent channels of the crystal could contribute a minor signal with little or no dichroism. Curve fitting included minor bands to represent unresolved contributions and calculation of the dichroic index from the fitted peak height of the high-spin band led to a dichroic index $\Pi=-0.65$ consistent with that calculated from the experimental absorbances at $2045 \mathrm{~cm}^{-1}$, and 

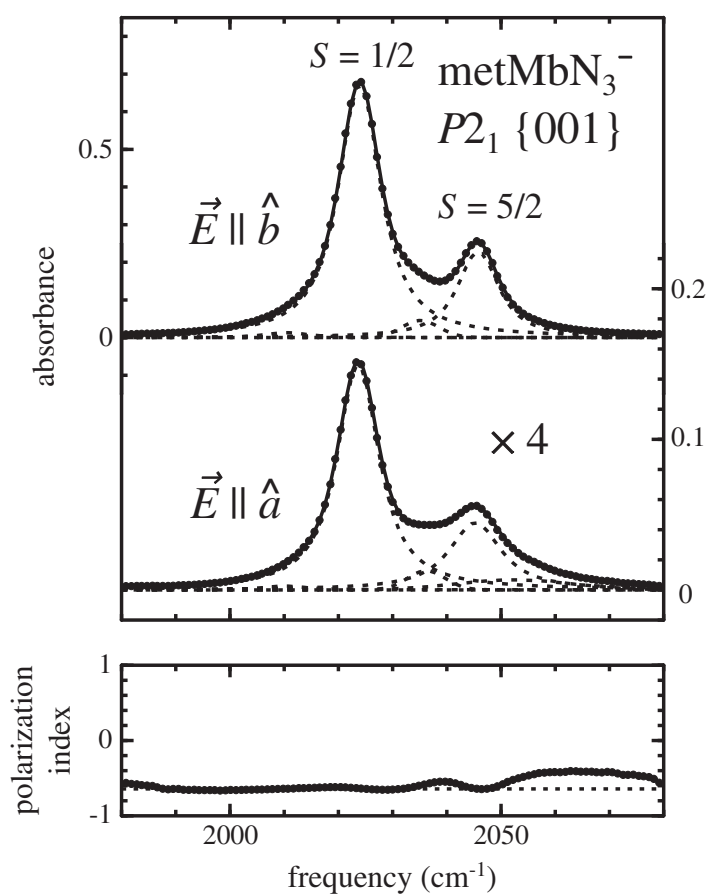

Fig. 10. Polarized infrared spectra recorded on the $\{001\}$ face of $P 2_{1}$ myoglobin crystals at ambient temperature reveal strongly polarized bands associated with antisymmetric stretching of the bound azide ligand. Prominent peaks at 2024 and $2045 \mathrm{~cm}^{-1}$ reflect $\mathrm{N}_{3}^{-}$bound to low-spin and high-spin hemes. The calculated dichroic index (Eq. 18) displayed in the lower panel has a nearly constant value $\Pi=-0.64 \pm 0.02$ (indicated by a dashed reference line) in the region of the low-spin peak, but exhibits larger variations at higher frequencies, associated with subtle polarization differences visible in the lineshape of the high-spin peak. Fits to Voigtian lineshapes (solid curve, with individual components and baseline indicated as dashed curves) include two minor bands in addition to the low- and high-spin bands. Spectra correspond to absorption changes after exposure of oxidized myoglobin crystals to $1 \mathrm{mg} / \mathrm{ml} \mathrm{NaN}$ dissolved in mother liquor. Data are recorded with polarization parallel to the $a$ - and $b$-axes, with the $a$-polarized data scaled up by a factor 4 for ease of lineshape comparison.

nearly identical to the value $\Pi=-0.64$ determined for the low-spin band.

\subsubsection{Uniaxial crystals}

In space groups of higher symmetry, averaging over molecules related by crystallographic symmetry reduces the orientational information available from experimental observations. The generating operations of trigonal, tetragonal, or hexagonal space groups include a single $n$-fold rotation with $n \geq 3$ in addition to translations and possibly inversions and 2-fold rotations. Symmetry compels alignment of the optic axis with the symmetry axis, whose direction we denote by the unit vector $\hat{u}$, and the averaging process described in the Appendix reveals that the absorbance

$\mathcal{A}_{1}=\frac{1}{2} \sum_{\alpha} 3 \epsilon_{\alpha} c d \frac{1}{m} \sum_{j=1}^{m}\left[1-\left(\hat{\mu}_{\alpha j} \cdot \hat{\mu}\right)^{2}\right]$

of the ordinary wave, for which $\hat{e}_{1} \perp \hat{u}$, is independent of propagation direction. The polarization $\hat{e}_{2}$ of the extraordinary wave depends on the direction of propagation, but the largest dichroism occurs when the propagation direction is normal to $\hat{u}$, so that $\hat{e}_{2}=\hat{k} \times \hat{e}_{1} \| \hat{u}$ and all symmetry-related molecules contribute identically to the absorbance

$\mathcal{A}_{2}=\sum_{\alpha} 3 \epsilon_{\alpha} c d \frac{1}{m} \sum_{j=1}^{m}\left(\hat{\mu}_{\alpha \mathrm{j}} \cdot \hat{\mu}\right)^{2}$.

For other polarization directions, absorption is determined by these two invariant quantities, according to Eq. (11).
The angular displacement $\delta_{\alpha j}$ of the transition dipole from the optic axis thus completely determines the polarization dependence of the absorption in uniaxial crystals (Fig. 9). Averaging over symmetryrelated molecules eliminates any information about the azimuthal angle in the plane orthogonal to $\hat{u}$, while all symmetry-related molecules contribute equivalently to the absorbance because the quantity $\left(\hat{\mu}_{\alpha j} \cdot \hat{u}\right)^{2}=\cos ^{2} \delta_{\alpha j}$ is invariant under all crystallographic symmetry operations. Thus, the optical anisotropy

$r_{\alpha}=\frac{\mathcal{A}_{2}-\mathcal{A}_{1}}{\mathcal{A}_{2}+2 \mathcal{A}_{1}}=\frac{1}{m} \sum_{j=1}^{m} P_{2}\left(\cos \delta_{\alpha j}\right)$.

determined from polarized absorption measurements performed with radiation perpendicular to the optic axis depends only on $\delta_{\alpha j}$. $\left(P_{2}(x)\right.$ is the second order Legendre polynomial.) For a single molecule in the asymmetric unit $(m=1)$, the measured anisotropy

$r_{\alpha}=P_{2}\left(\cos \delta_{\alpha j}\right)$

restricts the polar angle to one of two values $\delta_{\alpha}, \pi-\delta_{\alpha}$ that satisfy this experimental constraint.

Experimental constraints may not permit measurements normal to the optic axis. For example, proteins often crystallize as flat plates that facilitate measurement of radiation propagating parallel to the optic axis. In this case, Eq. (22) determines the absorbance, independent of polarization, and a spectrally resolved feature corresponding to mode $\alpha$ in the unpolarized crystal absorbance differs by a factor

$R_{\alpha}=\frac{3}{2} \frac{1}{m} \sum_{j=1}^{m} \sin ^{2} \delta_{\alpha j}$

from the absorbance $\mathcal{A}=\epsilon_{\alpha} c d$ of a randomly oriented molecular ensemble with the same concentration and pathlength.

Finally, in the presence of the four three-fold axes found in cubic space groups, Eq. (9) reduces to the isotropically averaged result

$\mathcal{A}=\sum_{\alpha} \epsilon_{\alpha} c d$

Measurements on cubic crystals are polarization-independent and yield no information on molecular orientation. On the other hand, Eq. (11) predicts no thickness-dependent spectral distortions for $\mathcal{A}_{1}=\mathcal{A}_{2}$. Thus, direct comparison with solution measurements provides a straightforward probe for any molecular changes in the crystalline state of cubic crystals.

\subsection{Molecular orientation}

Measurements on oriented single crystals constrain possible molecular orientations within a crystallographically determined laboratory coordinate system (Fig. 11). When expressed in a coordinate system fixed on the molecule, synthesis of this information either with stereochemical constraints or with other experimental measurements can generate true three-dimensional structural information. In its purest form, polarized measurements on multiple faces of a crystal or from different crystal forms can define the transition dipole orientation associated with a spectrally resolved vibrational mode $[62,64]$.

This is possible when X-ray crystallographic analysis defines the orientation of part of the molecule, such as the heme group in myoglobin, with sufficient precision to serve as the basis for a molecular coordinate system. The components of the dipole 


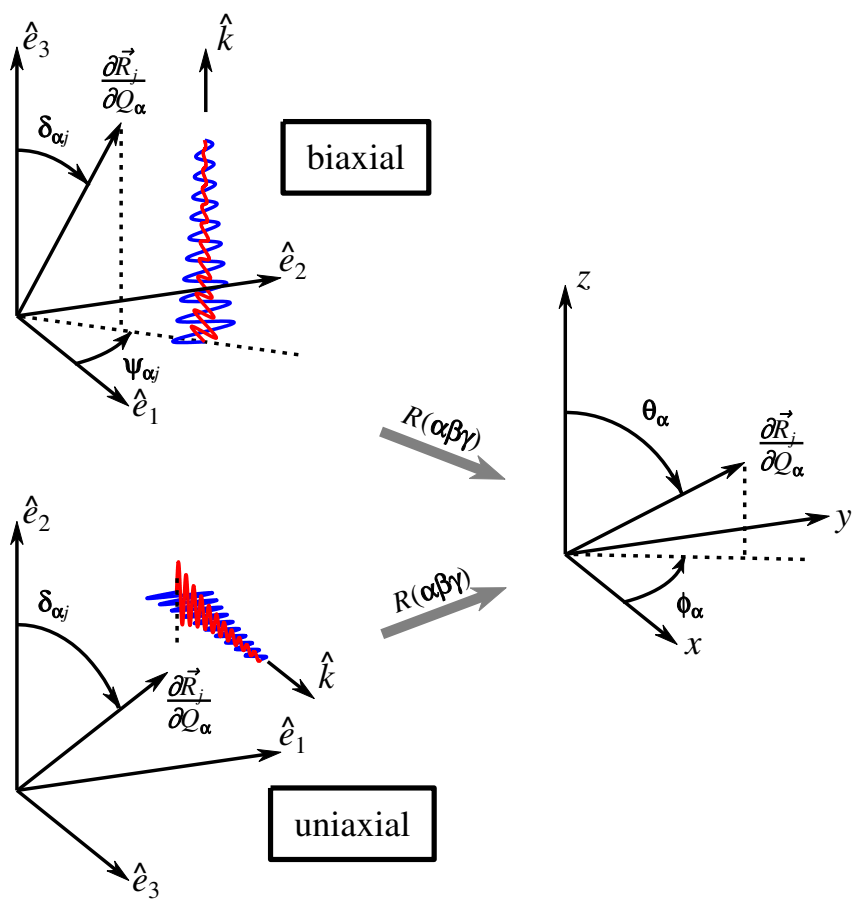

Fig. 11. The orientation of the infrared transition dipole $\partial \vec{R} / \partial Q_{\alpha}$, specified by a polar angle $\delta_{\alpha}$ and an azimuthal angle $\psi_{\alpha}$ in the crystallographic coordinate system, determines the absorbance of the two linearly polarized wave solutions to Maxwell's Equations (which also differ in wavelength). Because of orientational averaging, a uniaxial crystal exhibits dichroic absorption for light propagating perpendicular, but not parallel, to the high-symmetry direction $\hat{e}_{2}$. Euler angles $(\alpha \beta \gamma)$ relate the transition dipole orientation in the crystallographic system to its orientation in the molecular coordinate system, specified by angles $\left(\theta_{\alpha}, \varphi_{\alpha}\right)$.

orientation $\hat{\mu}_{m}=\left(\hat{\mu}_{x}, \hat{\mu}_{y}, \hat{\mu}_{z}\right)$ in the molecular coordinate system are related to the components $\hat{\mu}=\left(\hat{\mu}_{1}, \hat{\mu}_{2}, \hat{\mu}_{3}\right)$ in the laboratory coordinate system by the linear transformation

$\hat{\mu}_{m}=\tilde{R}(\alpha \beta \gamma) \cdot \hat{\mu}$

in which the rotation matrix

$\tilde{R}(\alpha \beta \gamma)=\left[\begin{array}{lll}\cos \gamma & \sin \gamma & 0 \\ -\sin \gamma & \cos \gamma & 0 \\ 0 & 0 & 0\end{array}\right]\left[\begin{array}{lll}\cos \beta & 0 & -\sin \beta \\ 0 & 1 & 0 \\ \sin \beta & 0 & \cos \beta\end{array}\right]\left[\begin{array}{lll}\cos \alpha & \sin \alpha & 0 \\ -\sin \alpha & \cos \alpha & 0 \\ 0 & 0 & 1\end{array}\right]$

is specified in terms of the Euler angles $(\alpha, \beta, \gamma)$ that rotate the crystal co-ordinate system $(1,2,3)$ into the molecular coordinate system $(x$, $y, z)$.

From experimentally determined values of $\delta$ or $\psi$, we wish to determine the polar and azimuthal angles $\theta$ and $\phi$ that determine the components of the dipole orientation

$\hat{\mu}_{m}=\left(\begin{array}{ll}\sin \theta \cos \phi, \sin \theta \sin \phi, \cos \theta\end{array}\right)$

in the chosen molecular coordinate system. Multiplying Eq. (28) on the left by the inverses of the first two matrices on the right-hand side of Eq. (29), we find the relations

$\sin \delta \cos (\psi-\alpha)=\cos \beta \sin \theta \cos (\phi+\gamma)+\sin \beta \cos \theta$,

$\sin \delta \sin (\psi-\alpha)=\sin \theta \sin (\phi+\gamma)$

$\cos \delta=-\sin \beta \sin \theta \cos (\phi+\gamma)+\cos \beta \cos \theta$ between the orientational angles defined in the two coordinate systems.

If the orientation $(\theta, \phi)$ of the transition dipole in the molecular frame and the Euler angles relating it to the laboratory system are both known, Eq. (33) or the ratio

$\tan (\psi-\alpha)=\frac{\sin \theta \sin (\phi+\gamma)}{\cos \beta \sin \theta \cos (\phi+\gamma)+\sin \beta \cos \theta}$

of Eqs. (31) and (32) then define the expected result of an experimental measurement of $\delta$ or $\psi$, respectively. Conversely, an experimental determination of a principal value $\psi_{\mathrm{p}}$ for $\psi$ restricts the orientation of the dipole to two branches of solutions with values of $\theta$ and $\phi$ satisfying the relations

$\tan \theta=\frac{ \pm \tan \left(\psi_{\mathrm{p}} \mp \alpha\right) \sin \beta}{\sin (\phi+\gamma) \mp \tan \left(\psi_{p} \mp \alpha\right) \cos \beta \cos (\phi+\gamma)}$

found by inverting Eq. (34) after inserting the possible values $\pm \psi_{p}$, $\pm\left(\pi-\psi_{p}\right)$ of $\psi$ consistent with the experimental measurement. Similarly, in uniaxial crystals, the determination of a principal value for $\delta$ requires that $\theta$ and $\phi$ satisfy

$\cos (\phi+\gamma)=\frac{\cos \beta \cos \theta \mp \cos \delta_{p}}{\sin \beta \sin \theta}$

to be consistent with the values $\delta_{\mathrm{p}}, \pi-\delta_{\mathrm{p}}$ allowed by experiment.

Fig. 12 illustrates orientations consistent with the IR dichroism measured for the $\mathrm{C}-\mathrm{O}$ stretching vibration of the heme ligand in oriented single crystals of $\mathrm{MbCO}[64,75]$ as points on the surface of a unit sphere aligned with the molecular coordinate system. Given a set $\{\alpha, \beta, \gamma\}$ of Euler angles relating the optical and molecular coordinate systems and an experimental value for $\psi_{\mathrm{p}}$ determined by the measured dichroism, Eq. (35) restricts allowed orientations to lie on two great circles that intersect at the propagation direction $\{\theta, \phi\}=\{\beta$, $\pi-\gamma\}$ of the radiation. The results of experimental measurements on multiple faces or crystal forms provide additional restrictions in the form of Eqs. (35) or (36) that can constrain the values of $\theta$ and $\phi$ to precisely defined regions. Alternatively, stereochemical constraints on bond angles may be invoked to help define allowed orientations. Experimental dichroism measurements alone were sufficient to define the three-dimensional orientation of the $\mathrm{C}-\mathrm{O}$ transition dipole in the case of MbCO [64] (see Section 6.3).

In some cases, a single measurement yields meaningful constraints on the value of $\theta$ or $\phi$ individually. For example, by minimizing the square of Eq. (35) as $\phi$ varies it is straightforward to demonstrate that

$\tan ^{2} \theta>\frac{\tan ^{2}\left(\psi_{p} \mp \alpha\right) \sin ^{2} \beta}{1+\tan ^{2}\left(\psi_{p} \mp \alpha\right) \cos ^{2} \beta}$

has a minimum value determined by $\psi_{\mathrm{p}}$. Similarly, differentiation of Eq. (36) with respect to $\phi$ and $\theta$, respectively, yields conditions

$\cos ^{2}(\theta \pm \beta)=\cos ^{2} \delta_{\mathrm{p}}$

and

$\cos ^{2}(\phi+\gamma)>\frac{\cos ^{2} \delta_{\mathrm{p}}-\cos ^{2} \beta}{1-\cos ^{2} \beta}$

respectively, for extremal values of $\theta$ and $\phi$, given an experimental value for $\delta_{\mathrm{p}}$. These Eqs. (38) and (39) provide the least information for the case $\cos ^{2} \beta>\cos ^{2} \delta_{\mathrm{p}}$, when there is no value of $\phi$ that is 


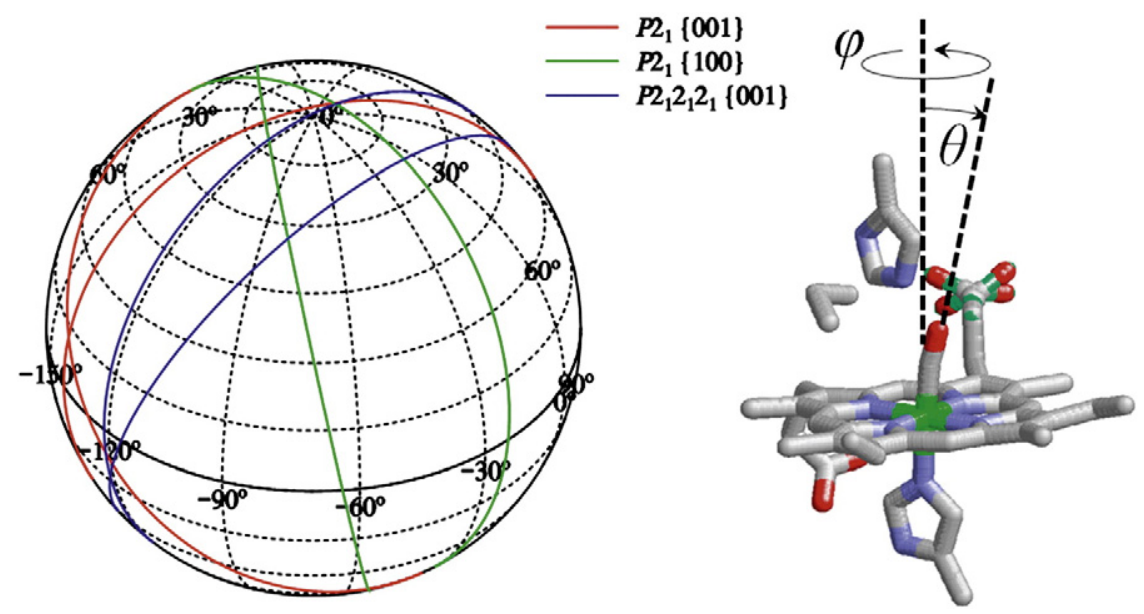

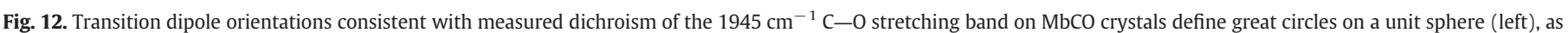

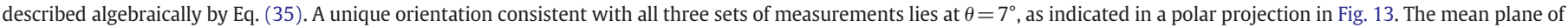

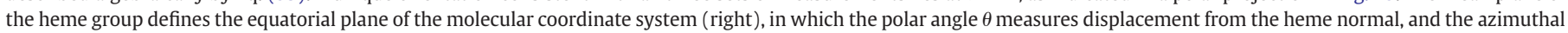

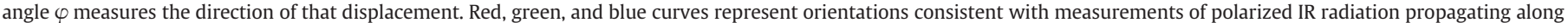

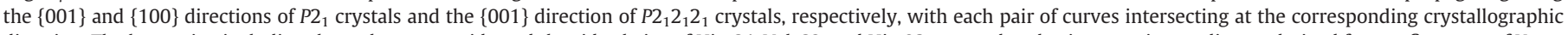

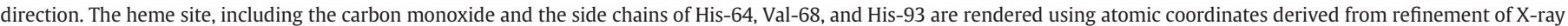
diffraction data collected at ambient temperature to $1.15 \AA ̊$ [66] (PDB ID: 1BZR).

inconsistent with the measured value of $\delta$, and $\theta$ may take any value in the ranges $\{0, \beta+\delta\}$ or $\{\pi-\beta-\delta, \pi\}$. When $\cos ^{2} \beta<\cos ^{2} \delta_{\mathrm{p}}$, Eq. (39) restricts the range of values of $\phi$, and $\theta$ must lie between the values $\beta \pm \delta$ or $\pi-(\beta \pm \delta)$.

\section{Applications}

\subsection{Spectroscopic comparison of proteins in the crystal and in solution}

How faithfully do structural models derived from modeling X-ray scattering from crystals represent the "actual structure" of a protein in solution? On one hand, this perennial question reflects some naivete. First, proteins constantly fluctuate among a large distribution of possible structures, which are often essential for their function. No single structure (or even the families of structures used to model NMR data) can adequately capture protein structural dynamics. Second, the cellular environment is highly crowded with other macromolecules and it is mistaken to assume that the dilute solutions typically used for biochemical studies provide an environment any less "unnatural" than the crystal lattice. Nevertheless, the value of an atomic structural model, no matter how detailed, is enhanced if it provides a reliable guideline for understanding investigations in dilute solution.

Spectroscopic comparisons provide an excellent means to probe possible structural differences between crystals and solutions, or among multiple crystalline forms. More generally, acknowledging the intrinsic variability of the protein conformation, spectroscopic comparisons may establish the conditions under which the structural model derived from X-ray diffraction actually applies in solution. Infrared spectroscopy is a particularly valuable approach because of its ability to probe multiple sites, including the protein backbone, side chains, ligands and prosthetic groups.

IR measurements on single crystals of myoglobin exploited reduced solvent absorption in the spectral "window" from 1800 to $2800 \mathrm{~cm}^{-1}$ to probe internal vibrations of heme ligands, including $\mathrm{N}_{3}^{-}$ and $\mathrm{CN}^{-}$[62] as well as $\mathrm{CO}[63,64,75]$. In all cases, peak frequencies observed for either $P 2_{1} 2_{1} 2_{1}$ or $P 2_{1}$ crystals of sperm whale myoglobin differed by no more than $2 \mathrm{~cm}^{-1}$ from those observed in solution. In $P 2_{1} \mathrm{MbCO}$ crystals, curve fitting analysis indicated a slight increase in the population of the $A_{3}$ conformation responsible for the shoulder near $1933 \mathrm{~cm}^{-1}$ with respect to solution [62]. However, the relative intensities of 2024 and $2046 \mathrm{~cm}^{-1}$ bands associated with asymmetric stretching of $\mathrm{N}_{3}^{-}$bound to low- and high-spin heme iron were unchanged [62].

These investigations also revealed significant conformational freedom for the protein in the crystalline phase. The population of the $1966 \mathrm{~cm}^{-1} \mathrm{~A}_{0}$ band increases as the $\mathrm{pH}$ of the solution bathing the crystal is reduced, becoming the dominant conformation at pH 4 (Fig. 7). Comparison with structures determined by refinement of X-ray diffraction data collected at variable $\mathrm{pH}$ establishes that the $\mathrm{A}_{0}$ band corresponds to an "open pocket" conformation of the crystal, where dihedral rotation of His64 about the $C_{\alpha}-C_{\beta}$ bond moves the imidazole side chain from the vicinity of the bound $\mathrm{CO}$ to a solvent-exposed location, opening a channel connecting the heme site to solvent [76]. Analogous pH-dependent Raman measurements on MbCO crystals associated the $491 \mathrm{~cm}^{-1} \mathrm{Fe}-\mathrm{CO}$ stretching frequency with the "open" conformation [77]. Titration of the $\mathrm{pH}$ demonstrates that the relative population of the "open" conformation in the crystal reversibly tracks that observed in solution $[63,77]$.

The similar spectral lineshapes observed in crystals and solutions further underline the conformational freedom of the protein in the crystalline environment. MbCO provides an excellent test case, because photon echo measurements provide a quantitative measure of the vibrational dephasing time that indicates a very narrow $\left(2.7 \mathrm{~cm}^{-1}\right)$ homogeneous line width [78]. As a result, the observed lineshape of the $\mathrm{C}-\mathrm{O}$ stretching vibration is primarily due to frequency variations in response to structural changes. The nearly identical lineshapes for the $\mathrm{C}-\mathrm{O}$ stretching band in crystal and solution phases are consistent with the idea that the crystal lattice allows individual heme sites to sample the full conformational distribution that they populate in solution (Fig. 7). Temperature-derivative IR measurements on polycrystalline samples of MbCO display a broad distribution of rates for geminate rebinding of photolyzed CO [45], confirming that these conformations are also distinct kinetically, as previously observed in frozen solutions [79]. Encapsulation of MbCO in a silica sol-gel reduces the vibrational dephasing of the $\mathrm{C}-\mathrm{O}$ stretch without noticeable effect on the lineshape [80], perhaps analogous to the effect of the crystalline environment.

Although crystallized proteins retain some conformational freedom, large conformational changes may be incommensurate with the crystalline lattice. In many cases, reactions with crystallized proteins cause crystals to disorder, crack, or dissolve. A classic example is the cracking of reduced hemoglobin $(\mathrm{Hb})$ crystals crystallized using high salt concentrations upon oxygen binding, presumably because the equilibrium ligated conformation (R-state) is incommensurate with 
the T-state lattice. In contrast, deoxyHbA crystallized at low salt concentrations using polyethylene glycol remain trapped in the lowaffinity T-state upon oxygen binding, permitting investigation of the intermediate ligated T-state structure [81,82].

Infrared measurements on single crystals of T-state human adult hemoglobin $(\mathrm{HbA})$ provided the opportunity to probe the $\mathrm{S}-\mathrm{H}$ vibrations of Cys $\alpha-104$ and Cys $\beta-112$, located at the $\alpha-\beta$ subunit interface, as well as the stretching vibration of $\mathrm{C}-\mathrm{O}$ at the heme sites [52]. The $\mathrm{S}-\mathrm{H}$ linewidths and frequencies in the deoxygenated crystal agree with those observed for deoxyHbA solution. On the other hand, saturation of the $P 2_{1} 2_{1} 2$ deoxyHbA crystals with $\mathrm{CO}$ leads to complete spectral depolarization, consistent with disruption of the crystal lattice, accompanied by $\mathrm{S}-\mathrm{H}$ and $\mathrm{C}-\mathrm{O}$ frequencies similar to those reported for the high-affinity R-state of HbACO in solution. However, $4 \% \mathrm{CO}$ saturation left the $\mathrm{S}-\mathrm{H}$ stretching region unaltered in frequency or polarization, consistent with retention of the T-state lattice. In this ligated $\mathrm{T}$ state, approximately $20 \%$ of the CO-ligated $\beta$-hemes adopt an alternate conformation with a $1967 \mathrm{~cm}^{-1} \mathrm{C}-\mathrm{O}$ frequency, similar to the "open pocket" conformation of MbCO [52].

Rather than using single crystals, several investigations arranged large numbers of protein crystals between $\mathrm{CaF}_{2}$ windows, to allow the collection of IR spectra of heme-bound CO without using an IR microscope. Early measurements on tetragonal R-state HbACO crystals revealed an increased width of the primary $\mathrm{C}-\mathrm{O}$ stretching band near $1951 \mathrm{~cm}^{-1}$ in comparison with HbACO solution, as well as increased population of a minor conformation with a $1969 \mathrm{~cm}^{-1}$ frequency [42]. Curve-fitting analysis resolved the primary band into two subbands of comparable area, and the authors concluded that the frequency of $\mathrm{CO}$ bound to the $\alpha$ - and $\beta$-hemes increased by 1 and $5 \mathrm{~cm}^{-1}$, respectively, upon crystallization [42]. In contrast, the frequencies of CO bound to the catalytic site of bovine cytochrome $c$ oxidase differed by less than $0.2 \mathrm{~cm}^{-1}$ from the solution frequencies [43].

However, IR measurements on polycrystalline arrays of $\mathrm{MbCO}$ have reported variable results $[41,45]$. Different preparations of polycrystalline sperm whale $\mathrm{MbCO}$ displayed significant variations in the relative population of the $\mathrm{A}_{3}$ conformation at ambient temperature, which were attributed to varying levels of $\mathrm{CO}$ saturation and partial oxidation of the heme [41]. In all cases, the population of the $A_{3}$ conformation was significantly larger than observed either in parallel solution measurements [41] or in later measurements on single crystals of MbCO [62-64,75]. In contrast, measurements of polycrystalline arrays of $\mathrm{MbCO}$ at low temperatures revealed much smaller spectral differences with respect to solution measurements at the same temperature [45].

Although the reason for the variable results is unclear, they underline the need for caution in preparation of polycrystalline samples. The partial heme oxidation reported for some samples [41] raises the possibility that prolonged exposure to atmosphere may also have led to drying of the crystals. The experimental description for the low temperature measurements describes "crushing" the crystals between $\mathrm{CaF}_{2}$ windows [45], and this may prevent optical distortion due to variations in sample thickness that may also have influenced the earlier measurements [41]. The use of single crystals usually eliminates the need for mechanical manipulation of crystals, avoiding uncertainties about the effects of mechanical strain.

A number of approaches have been taken to extend infrared measurements on single crystals into the $1000-1800 \mathrm{~cm}^{-1}$ region. This region is highly informative, because numerous signals from the protein backbone and side chains appear in this region. The strong solvent absorption requires very short pathlengths, but results have been obtained on crystals of several photoactive proteins.

Nucleation between $\mathrm{CaF}_{2}$ windows separated by a $6.5 \mu \mathrm{m}$ teflon spacer led to crystallization of photosynthetic reaction centers from Rhodopseudomonas viridis in the $P 4_{1} 2_{1} 2$ space group with an extremely large aspect ratio $(1 \mathrm{~mm} \times 0.5 \mathrm{~mm} \times 6.5 \mu \mathrm{m})$, permitting IR difference measurements below $1800 \mathrm{~cm}^{-1}$ [38]. Electronic absorption measure- ments confirmed that steady-state $900-1000$ nm illumination populated the charge-separated $\mathrm{P}^{+} \mathrm{Q}^{-}$state, in which the primary donor is oxidized and the quinone acceptor is reduced. Strong absorption by the solvent $\mathrm{HOH}$ bend at $1640 \mathrm{~cm}^{-1}$ and by phosphate buffer and ammonium sulfate in the mother liquor from 1050 to $1150 \mathrm{~cm}^{-1}$ increased noise levels in these regions. Nevertheless, infrared difference spectra with respect to the initial state at $300 \mathrm{~K}$ exhibited several features that were reproducible in measurements on different crystals. The frequencies and relative intensities also showed good agreement with measurements on reaction centers reconstituted into lipid vesicles [83]. These steady-state difference measurements indicate the successful population of the $\mathrm{P}^{+} \mathrm{Q}^{-}$photocycle intermediate in the crystalline state.

Steady-state IR measurements on other membrane bound photoactive proteins support the retention of photoactivity in the crystalline environment. For example, infrared spectral differences between illuminated and non-illuminated sensory rhodopsin II in the crystalline state and when reconstituted in liposomes compared well over much of the $1000-1800 \mathrm{~cm}^{-1}$ region. However, reduced intensities in the strongly absorbing amide I and amide II regions were attributed to smaller conformational changes in the $P 2_{1} 2_{1} 2$ crystal [84].

Time-resolved infrared and resonance Raman spectroscopy on hexagonal bR microcrystals revealed spectroscopic evidence for photoinduced spectroscopic changes similar to those observed in the native purple membrane [44]. A more detailed comparison of timeresolved IR measurements on single hexagonal bR crystals with parallel measurements on purple membranes confirmed the conclusion that the protein remains a functional proton pump in the crystalline phase, although some small frequency shifts were observed [40]. However, the photocycle kinetics were clearly altered in the crystal. In particular, the $\mathrm{L} \rightarrow \mathrm{M}$ transition was accelerated, while later steps in the photocycle were retarded in the crystal. Possible reasons suggested for the perturbed kinetics include the reduced water content and altered lipid composition. Further evidence that kinetics differ in the crystalline phase is the appearance of spectral features of both $\mathrm{L}$ and $\mathrm{M}$ intermediates in IR difference spectra induced by illumination of bR crystals at $230 \mathrm{~K}$ [117], while analogous measurements found $95 \%$ population of the M state in illuminated purple membrane at the same temperature [118]. Infrared measurements guided the development of protocols designed to trap specific photocycle intermediates for X-ray crystallographic studies [85].

Time-resolved infrared measurements of the photocycle of photoactive yellow protein (PYP) crystals reveal spectroscopic changes significantly smaller than those observed in solution [46]. PYP is a bacterial photosensor, and these results have important implications for understanding the mechanism of photoreception. In particular, formation of the signaling intermediate in the solution photocycle appears to involve conformational changes significantly larger than those observed in the crystalline state [86,87].

All of these measurements are consistent with minimal perturbation of the conformational equilibrium of the protein by the crystal lattice. However, the kinetics of conformational interconversion are significantly perturbed in the crystalline phase. Time-resolved X-ray crystallography provides a detailed picture of structural dynamics, but lattice constraints can impede the kinetics and perturb the structure of intermediate conformations. Quantitative analysis of time-resolved $\mathrm{X}$-ray crystallography on myoglobin crystals reveals that the fraction of photolyzed CO molecules that rebind geminately to the same heme following photolysis is larger than in solution [88]. Qualitatively similar effects are observed in highly viscous glycerol/water solutions, which impede the motion of the distal histidine that opens the primary channel for ligand escape from the protein [89]. Together with observation of transient electron density attributed to CO occupancy of the $\mathrm{Xe}_{1}$ pocket on the proximal side of the heme [88], these results are consistent with lattice-induced suppression of $\mathrm{CO}$ escape via the classical pathway gated by His 64 . It remains to be established whether 
the $\mathrm{Xe}_{1}$ pocket plays a significant role for $\mathrm{CO}$ migration in aqueous solution.

\subsection{Spectroscopic identification of molecular species generated during $X$-ray exposure of protein crystals}

Even when protein structure is unaffected by the crystallization process itself, the prolonged X-ray exposures required to collect a complete set of high-resolution diffraction data can lead to chemical and structural modifications [9-11,90]. At high doses, scattering intensities decrease, with $30 \mathrm{MGy}(1 \mathrm{~Gy}=1 \mathrm{~J} / \mathrm{kg})$ recommended as a practical limit for "typical macromolecular crystallography experiments" [91] ( $1 \mathrm{~Gy}=1 \mathrm{~J} / \mathrm{kg})$. However, significant photoreduction of metal sites takes place at significantly lower doses, and visible absorption measurements can monitor this process in favorable cases [8,17-19].

Infrared spectroscopy can probe irradiated crystals that lack a visible chromophore, providing a means to monitor and characterize dose-dependent modifications of the polypeptide. Infrared measurements on butyrylcholinesterase (BChE) crystals, following irradiation with X-rays at $100 \mathrm{~K}$, reveal spectral contributions of molecular species resulting from radiation exposure, including bands at 2340 , 2165 and $2136 \mathrm{~cm}^{-1}$ (Fig. 5). We previously reported $\mathrm{CO}_{2}$ bands near $2340 \mathrm{~cm}^{-1}$ resulting from UV-induced decarboxylation of green fluorescent protein, coupled with electron density consistent with photogenerated $\mathrm{CO}_{2}[22]$. Although decarboxylation of Asp and Glu residues has often been noted as a consequence of X-ray exposure, electron density attributable to the resulting $\mathrm{CO}_{2}$ has been reported less often, perhaps due to its high mobility [47]. The strong $2340 \mathrm{~cm}^{-1}$ band observed in irradiated BChE crystals (Fig. 5) is consistent with generation of $\mathrm{CO}_{2}$. Weaker spectral features resulting from irradiation remain to be identified, although the $2136 \mathrm{~cm}^{-1}$ band may reflect the generation of $\mathrm{CO}$. Note that irradiation of the cryosolvent appears to generate some of the same products, but with differing relative populations (Fig. 5). Irradiation of selected nonaqueous liquids at room temperature generated gaseous products including $\mathrm{H}_{2}, \mathrm{CO}$, and $\mathrm{CH}_{4}$, and $\mathrm{CO}_{2}$ production was reported only for a yeast suspension [92].

\subsection{Exploiting molecular orientation in oriented single crystals}

Fraser's investigations of DNA structure in the early 1950s are a notable early application of polarized infrared absorption measurements to an important biological problem. Although single crystals were not available, polarization measurements on aligned DNA fibers clearly demonstrated that the bases were oriented perpendicular to the fiber axis [32]. Fraser used these results, in conjunction with other measurements and physical reasoning, to propose a structural model for DNA that captured important elements of the structure that would shortly be reported by Crick and Watson, featuring hydrogen bonded bases stacked along the axis of a helical structure with phosphate groups on the outside. The faulty element, a detail with profound biological ramifications, was the presence of three intertwined chains, rather than two. Although cited in the third paragraph of the famous Watson-Crick publication [93], Fraser's manuscript was never published at the time, appearing many years after the fact as an historical note [94].

Fraser continued to apply infrared dichroism for many years in studies on fibrous proteins [95], and this work proved seminal for later infrared investigations of oriented macromolecules. For example, polarized infrared measurements of membrane proteins probe the average orientation of helical elements with respect to the membrane normal $[61,96,97]$. The dichroism of the partially oriented molecular ensemble created by photochemical reaction initiation with polarized visible light also yields molecular information, although accurate quantitative interpretation requires careful determination of the orientational distribution $[30,98,99]$.

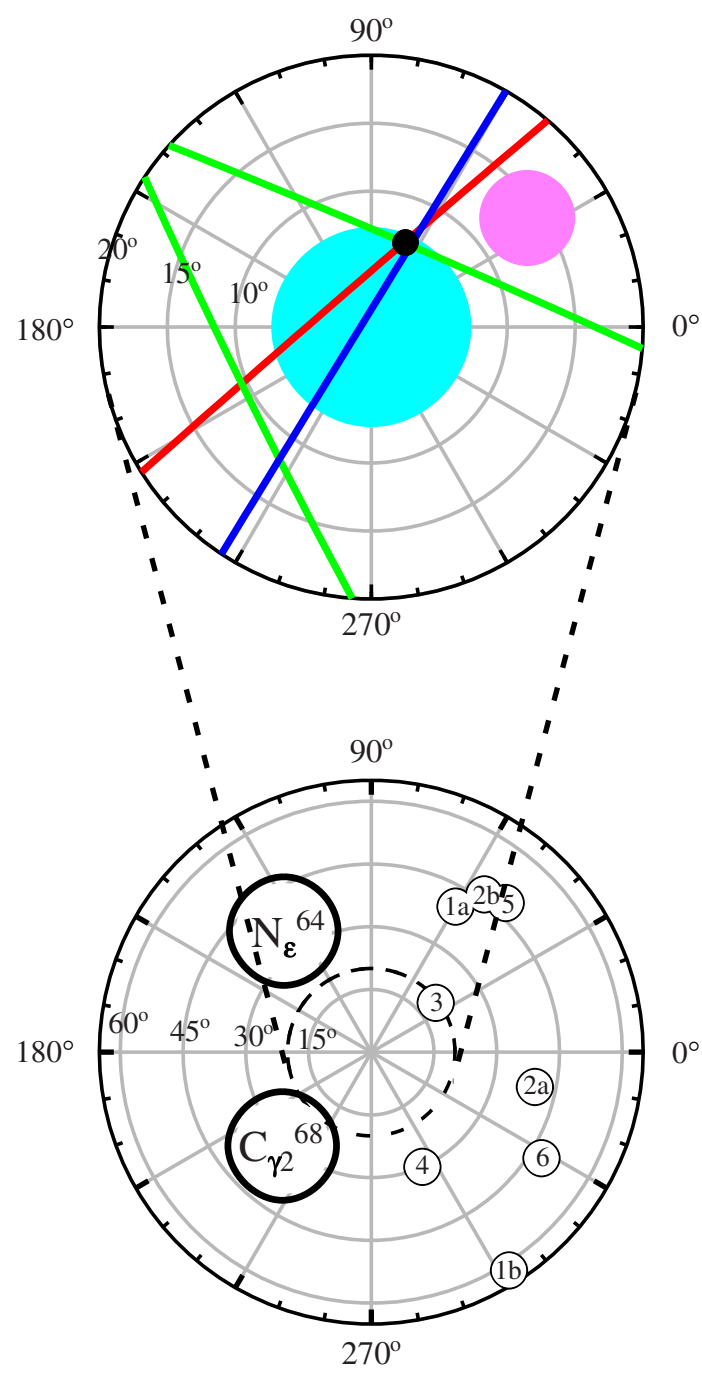

Fig. 13. Polar projections summarize experimental information on the orientation of $\mathrm{CO}$ bound to the heme group of myoglobin at ambient temperature. Early structures derived from refinement of X-ray diffraction data (below) included a wide range of orientations, all indicating large angular displacements of the CO from its energetically preferred orientation perpendicular to the heme, which would minimize steric interactions with the side chains His-64 and Val-68. Later measurements (above) include polarized infrared measurements on $P 2_{1}$ and $P 2_{1} 2_{1} 2_{1}$ MbCO crystals [75] (same color code as Fig. 12), which locate the orientation of the $\mathrm{C}-\mathrm{O}$ transition dipole $7^{\circ}$ from the heme normal (black point) [64] and visible-infrared photoselection measurements consistent with transition dipole orientations within $7^{\circ}$ of the normal (cyan region) $[99,100]$. A high-resolution X-ray structure (pale purple) [66] identifies a C-O bond orientation (magenta) much closer to the heme normal than earlier structures refined at lower resolution. The polar angle $\theta$ indicates the angular displacement of the $\mathrm{C}-\mathrm{O}$ bond vector from the heme normal (at center), while the azimuthal angle $\varphi$ indicates the direction of the displacement (see Fig. 12). For orientation, the lower plot displays directions from the heme iron to the nearest heavy atom of His-64 and Val-68.

The much higher degree of molecular orientation in protein crystals makes polarized infrared measurements particularly promising. Myoglobin $(\mathrm{Mb})$ served as a favorable case for developing and illustrating the capabilities of infrared protein crystallography [62-64,75]. Mb crystallizes relatively easily in space groups with symmetry low enough to exhibit dichroism, the vibrations of many heme ligands can be probed in relatively thick crystals, and the presence of a single molecule in the asymmetric unit simplifies the interpretation of polarized measurements.

Polarized IR measurements on MbCO crystals demonstrated that conformations having distinct $\mathrm{C}-\mathrm{O}$ vibrational frequencies nevertheless exhibited CO orientations within a few degrees of each other [75]. Moreover, the projected orientations of the $\mathrm{C}-\mathrm{O}$ transition 
dipole for the primary band at $1945 \mathrm{~cm}^{-1}$ and the heme normal were within $5^{\circ}$ for three independent directions in two crystallographic systems. Synthesis of this information in a common molecular coordinate system identified a unique orientation consistent with all measurements (Fig. 12).

In contrast with X-ray structures available at the time, which showed the $\mathrm{C}-\mathrm{O}$ bond displaced from its energetically preferred orientation normal to the heme by amounts ranging from $40^{\circ}$ to $60^{\circ}$ (lower panel of Fig. 13), infrared crystallography found the $\mathrm{C}-\mathrm{O}$ transition dipole displaced only $7^{\circ}$ from the heme normal $[63,64]$. Infrared photoselection measurements on $\mathrm{MbCO}$ solutions confirmed a C-O displacement of $7^{\circ}$ or less $[99,100]$. Other spectroscopic measurements were also consistent with reduced CO displacements [101]. With improving resolution, smaller FeCO distortions ultimately appeared in X-ray structural models as well [66,102]. Isotropic refinement of partially oxidized crystals may have contributed to the highly distorted FeCO unit in earlier structural models [103].

Based on DFT calculations, Spiro and Kozlowski suggested that the $\mathrm{C}-\mathrm{O}$ transition dipole lies closer to the heme normal than the $\mathrm{C}-\mathrm{O}$ bond [104]. This is plausible, and qualitatively accounts for the small remaining discrepancy between infrared and X-ray crystallographic results (Fig. 13). However, the predicted noncoincidence of the transition dipole and bond orientations still awaits a more direct experimental test. It may be significant that the transition dipole of the asymmetric azide stretch determined for metMbN ${ }_{3}^{-}$using infrared crystallography also exhibits a smaller displacement from the heme normal than the axis of the $\mathrm{N}_{3}^{-}$ligand in the X-ray structure [62].

These measurements contributed to the evolving understanding of protein recognition of diatomic molecules. Specific recognition of $\mathrm{O}_{2}$, $\mathrm{NO}$, and $\mathrm{CO}$ is essential because they play important but distinct physiological roles in cellular metabolism and in molecular signaling. When bound to heme, they exhibit significant variations in geometry and charge distribution that can be recognized by a protein, although the isolated diatomics are nearly indistinguishable by these criteria [105]. Protein distortion of the heme-CO complex from its energetically favorable geometry with CO normal to the heme plane could discriminate in favor of $\mathrm{O}_{2}$, which binds at an angle to the heme normal. In fact, calculations predict a $1-4 \mathrm{~kJ} / \mathrm{mol}$ cost associated with a 0.3-0.6 $\AA$ off-axis displacement of the oxygen atom [106], which, augmented by the energetic cost of distorting the protein matrix [63], could be physiologically significant. However, differences in charge distribution are now believed to play the primary role in favoring the binding of $\mathrm{O}_{2}$ over $\mathrm{CO}$ in myoglobin and hemoglobin. Specifically, transfer of electron density from the heme $\mathrm{Fe}$ to $\mathrm{O}_{2}$ upon heme binding favors the formation of a strong hydrogen bond with the distal histidine found in these oxygen carriers [106], while hydrogen bonding with CO [107] is significantly weaker.

Dichroic IR measurements of the $\mathrm{S}-\mathrm{H}$ band in single crystals of human adult hemoglobin ( $\mathrm{HbA}$ ) crystallized in the $P 2_{1} 2_{1} 2$ space group as the low-oxygen-affinity $\mathrm{T}$ state successfully isolated spectrally unresolved contributions from Cys $\alpha 104$ and Cys $\beta 112$ based on their distinct polarization properties [52]. Comparison of the polarization results with the structural model derived from X-ray diffraction measurements allowed assignment of 2558 and $2564 \mathrm{~cm}^{-1} \mathrm{~S}-\mathrm{H}$ vibrational frequencies to Cys $\alpha 104$ and Cys $\beta 112$, respectively. Specifically, the measured polarization indices were consistent with hydrogen bonding to the backbone carbonyls of Leu $\alpha 100$ and Asn $\beta 108$, respectively, supporting a proposed motif for hydrogen bonding of cysteine residues in $\alpha$-helices [108]. Synergistic application of infrared and X-ray crystallography to directly evaluate hydrogen bonding partners is intriguing, since macromolecular crystals are only rarely refined to a resolution that allows identification of hydrogen positions with confidence, so that hydrogen bonding must usually be inferred from indirect stereochemical reasoning.

Polarized IR measurements of the $\mathrm{C}-\mathrm{O}$ band following partial saturation of the HbACO crystal with $\mathrm{CO}$ probed the structure of the heme sites [52]. In particular, the dichroism of the primary C—O stretching band indicated comparable occupancy of the $\alpha$ - and $\beta$-hemes, not an obvious result in light of differing $\mathrm{O}_{2}$ affinities for the two sites [81,109]. Interestingly, polarization measurements associate the structural perturbation responsible for appearance of the secondary $1967 \mathrm{~cm}^{-1}$ band in the ligated $\mathrm{T}$ state with the $\beta$-heme [52].

\section{Summary and prospects}

Spectral comparisons with proteins in solution have been the most common application for infrared measurements on protein crystals. Although the equilibrium structure appears to be unaffected by crystallization in many cases, the time scale of conformational changes is often altered. This may complicate the interpretation of time-resolved measurements, but can also be exploited to trap metastable intermediate structures.

Polarized IR measurements have been fewer in number, but illustrate the opportunities for exploiting molecular orientation in the crystalline phase. In favorable cases, polarization measurements on multiple crystal forms can reveal the three-dimensional orientation of transition dipoles associated with single bonds. More commonly, infrared measurements complement X-ray crystallographic investigations, for example, by identifying the vibrational signatures of individual residues or by distinguishing among possible hydrogen bonding arrangements.

Infrared protein crystallography will realize its full potential with the extension of polarized IR measurements to the information-rich spectral region below $1800 \mathrm{~cm}^{-1}$. We are aware of two measurements demonstrating this possibility. In one case, a spectroelectrochemical cell enabled measurement of polarized IR spectral differences between oxidized and reduced states of a very thin crystal of cytochrome $c$ [39]. In the other case, measurements using the collimated IR radiation available at a synchrotron beamline allowed the measurement of the data on photosystem II crystals presented in Fig. 4. Previous measurements on single crystals below $1800 \mathrm{~cm}^{-1}$ have not reported polarization results. In addition to having dimensions that allow high transmission over the diameter of the available IR beam, crystals must have a favorable symmetry. Crystals with tetragonal or hexagonal space groups may develop plate-like habits that are favorable for infrared measurements along the high symmetry axis, but exhibit no optical anisotropy as a consequence of the high symmetry.

\section{Acknowledgements}

We acknowledge generous financial support from a National Science Foundation award CHE-1026369 (JTS) and from the Royal Society and from BBSRC award BB/H004238/1 (JJvT). Use of the National Synchrotron Light Source, Brookhaven National Laboratory, was supported by the U.S. Department of Energy, Office of Science, Office of Basic Energy Sciences, under Contract No. DE-AC02-98CH10886. We thank Prof. James Barber for supplying photosystem II crystals for this study. We thank Florian Nachon and Jacques-Philippe Colletier for purifying and crystallizing human butyrylcholinesterase. We are grateful to the ESRF for beam-time under long-term projects MX551 and MX666 (radiationdamage BAG).

\section{Appendix A}

Here we briefly demonstrate the calculation of the average

$$
\left\langle(\hat{\mu} \cdot \hat{e})^{2}\right\rangle=\frac{1}{n} \sum_{k=1}^{n}\left(\tilde{O}_{k} \cdot \hat{\mu} \cdot \hat{e}\right)^{2}
$$

of $(\hat{\mu} \cdot \hat{\mathrm{e}})^{2}$ over $n$ symmetry operations $\tilde{O}_{k}$. Each operator can be decomposed into products of translations, rotations, and inversions 
that generate the space group of the crystal. The quantity $(\hat{\mu} \cdot \hat{e})^{2}$ is clearly invariant under translations and inversions, and it is sufficient to consider the most symmetric rotational axis. For light polarized parallel to an $n$-fold axis, $(\hat{\mu} \cdot \hat{e})^{2}$ is again clearly invariant, leading to

$\left\langle(\hat{\mu} \cdot \hat{e})^{2}\right\rangle=(\hat{\mu} \cdot \hat{e})^{2}$

To consider the result for an $n$-fold rotation axis perpendicular to $\hat{e}$, we write the components of $\hat{\mu}$ in a coordinate system whose $z$ - and $x$-axes lie along the rotation axis and $\hat{e}$, respectively. We then express the average

$\left\langle(\hat{\mu} \cdot \hat{e})^{2}\right\rangle=\frac{1}{n} \sin ^{2} \theta \sum_{k=1}^{n} \cos ^{2}\left(\phi+\frac{2 \pi k}{n}\right)$

in terms of the polar and azimuthal angles $\theta$ and $\phi$. Trigonometric identities allow us to rewrite this as

$\left\langle(\hat{\mu} \cdot \hat{e})^{2}\right\rangle=\frac{1}{2 n} \sin ^{2} \theta\left[n+\cos 2 \phi \sum_{k=1}^{n} \cos \frac{4 \pi k}{n}-\sin 2 \phi \sum_{k=1}^{n} \sin \frac{4 \pi k}{n}\right]$.

For the case $n=2$, the right-hand side of Eq. (43) reduces to $(\hat{\mu} \cdot \hat{e})^{2}=$ $\sin ^{2} \theta \cos ^{2} \phi$, as when $\hat{e}$ is parallel to the axis. For $n \geq 3$, we easily evaluate the sums remaining in Eq. (43) as the real and imaginary parts of

$\sum_{k=1}^{n} e^{i 4 \pi k / n}=\left(1-e^{i 4 \pi}\right) \sum_{k=1}^{\infty} e^{i 4 \pi k / n}=\frac{1-e^{i 4 \pi}}{1-e^{i 4 \pi / n}}=0$,

concluding that

$\left\langle(\hat{\mu} \cdot \hat{e})^{2}\right\rangle=\frac{1}{2} \sin ^{2} \theta=\frac{1}{2}\left(1-(\hat{\mu} \cdot \hat{u})^{2}\right)$

for light polarized perpendicular to the direction û of an $n$-fold axis. The other situation of interest here occurs in cubic crystals, where the natural co-ordinate system is one in which each axis bisects a pair of three-fold rotations, such that the $2 \pi / 3$ rotation simply permutes the $x-, y$-, and $z$-components of $\hat{\mu}$. For light polarized along one of these axes, then,

$\left\langle(\hat{\mu} \cdot \hat{e})^{2}\right\rangle=\frac{1}{3}\left(\hat{\mu}_{x}^{2}+\hat{\mu}_{y}^{2}+\hat{\mu}_{z}^{2}\right)=\frac{1}{3}$.

\section{References}

[1] P.R. Carey, Raman crystallography and other biochemical applications of Raman microscopy, Annu. Rev. Phys. Chem. 57 (2006) 527-554.

[2] T.D. la Mora-Rey, C.M. Wilmot, Synergy within structural biology of single crystal optical spectroscopy and X-ray crystallography, Curr. Opin. Struct. Biol. 17 (2007) 580-586.

[3] A. Royant, P. Carpentier, J. Ohana, J. McGeehan, B. Paetzold, M. Noirclerc-Savoye, X Vernède, V. Adam, D. Bourgeois, Advances in spectroscopic methods for biological crystals. 1. Fluorescence life-time measurements, J. Appl. Cryst. 40 (2007) 1105-1112

[4] P. Carpentier, A. Royant, J. Ohana, D. Bourgeois, Advances in spectroscopic methods for biological crystals. 2. Raman spectroscopy, J. Appl. Cryst. 40 (2007) 1113-1122.

[5] M.A. Hough, S.V. Antonyuk, R.W. Strange, R.R. Eady, S.S. Hasnain, Crystallography with online optical and X-ray absorption spectroscopies demonstrates an ordered mechanism in copper nitrite reductase, J. Mol. Biol. 378 (2008) 353-361.

[6] L.M. Utschig, S.D. Chemerisov, D.M. Tiede, O.G. Poluektov, Electron paramagnetic resonance study of radiation damage in photosynthetic reaction center crystals, Biochemistry 47 (2008) 9251-9257.

[7] A.R. Pearson, R.L. Owen, Combining X-ray crystallography and single-crystal spectroscopy to probe enzyme mechanisms, Biochem. Soc. Trans. 37 (2009) 378-381.

[8] J. McGeehan, R.B.G. Ravelli, J.W. Murray, R.L. Owen, F. Cipriani, S. McSweeney, M. Weik, E.F. Garman, Colouring cryo-cooled crystals: online microspectrophotometry, J. Synchrotron Radiat. 16 (2009) 163-172.

[9] M. Weik, R.B. Ravelli, G. Kryger, S. McSweeney, M.L. Raves, M. Harel, P. Gros, I. Silman, J. Kroon, J.L. Sussman, Specific chemical and structural damage to protein produced by synchrotron radiation, Proc. Natl Acad. Sci. USA 97 (2000) 623-628.
[10] R.B. Ravelli, S.M. McSweeney, The "fingerprint" that X-rays can leave on structures, Structure 8 (2000) 315-328.

[11] W.P. Burmeister, Structural changes in a cryo-cooled protein crystal owing to radiation damage, Acta Cryst. D 56 (2000) 328-341.

[12] E.F. Garman, Radiation damage in macromolecular crystallography: what is it and why should we care? Acta Cryst. D 66 (2010) 339-351.

[13] N. Engler, A. Ostermann, A. Gassmann, D.C. Lamb, V.E. Prusakov, J. Schott, R. Schweitzer-Stenner, F.G. Parak, Protein dynamics in an intermediate state of myoglobin: optical absorption, resonance Raman spectroscopy, and x-ray structure analysis, Biophys. J. 78 (2000) 2081-2092.

[14] J. Yano, J. Kern, K.-D. Irrgang, M.J. Latimer, U. Bergmann, P. Glatzel, Y. Pushkar, J. Biesiadka, B. Loll, K. Sauer, J. Messinger, A. Zouni, V.K. Yachandra, X-ray damage to the $\mathrm{Mn}_{4} \mathrm{Ca}$ complex in single crystals of photosystem II: a case study for metalloprotein crystallography, Proc. Natl Acad. Sci. USA 102 (2005) 12047-12052.

15] T. Beitlich, K. Kühnel, C. Schulze-Briese, R.L. Shoeman, I. Schlichting, Cryoradiolytic reduction of crystalline heme proteins: analysis by UV-vis spectroscopy and X-ray crystallography, J. Synchrotron Radiat. 14 (2007) 11-23.

16] A.R. Pearson, R. Pahl, E.G. Kovaleva, V.L. Davidson, C.M. Wilmot, Tracking X-rayderived redox changes in crystals of a methylamine dehydrogenase/amicyanin complex using single-crystal UV/vis microspectrophotometry, J. Synchrotron Radiat. 14 (2007) 92-98.

17] J. Yi, A.M. Orville, J.M. Skinner, M.J. Skinner, G.B. Richter-Addo, Synchrotron Xray-induced photoreduction of ferric myoglobin nitrite crystals gives the ferrous derivative with retention of the O-bonded nitrite ligand, Biochemistry 49 (2010) 5969-5971.

[18] Y.T. Meharenna, T. Doukov, H. Li, S.M. Soltis, T.L. Poulos, Crystallographic and single-crystal spectral analysis of the peroxidase ferryl intermediate, Biochemistry 49 (2010) 2984-2986.

[19] H.-P. Hersleth, K.K. Andersson, How different oxidation states of crystalline myoglobin are influenced by X-rays, Biochim. Biophys. Acta (2010).

[20] D. Bourgeois, M. Weik, Kinetic protein crystallography: a tool to watch proteins in action, Cryst. Rev. 15 (2009) 87-118.

[21] M. Towrie, D.C. Grills, J. Dyer, J.A. Weinstein, P. Matousek, R. Barton, P.D. Bailey, N. Subramaniam, W.M. Kwok, C. Ma, D. Phillips, A.W. Parker, M.W. George, Development of a broadband picosecond infrared spectrometer and its incorporation into an existing ultrafast time-resolved resonance Raman, UV/visible, and fluorescence spectroscopic apparatus, Appl. Spec. 57 (2003) 367-380.

[22] J.J. van Thor, G.Y. Georgiev, M. Towrie, J.T. Sage, Ultrafast and low barrier motions in the photoreactions of the green fluorescent protein, J. Biol. Chem. 280 (2005) 33652-33659.

[23] J.J.van Thor, G. Zanetti, K.L. Ronayne, M. Towrie, Structural events in the photocycle of green fluorescent protein, J. Phys. Chem. B 109 (2005) 16099-16108.

[24] J.J. van Thor, J.T. Sage, Charge transfer in green fluorescent protein, Photochem. Photobiol. Sci. 5 (2006) 597-602.

[25] J.J. van Thor, K.L. Ronayne, M. Towrie, Formation of the early photoproduct lumi$\mathrm{R}$ of cyanobacterial phytochrome Cph1 observed by ultrafast mid-infrared spectroscopy, J. Am. Chem. Soc. 129 (2007) 126-132.

[26] M.D. Fayer, Dynamics of liquids, molecules, and proteins measured with ultrafast 2D IR vibrational echo chemical exchange spectroscopy, Annu. Rev. Phys. Chem. 60 (2009) 21-38.

[27] P. Hamm, Femtosecond IR pump-probe spectroscopy of nonlinear energy localization in protein models and model proteins, J. Biol. Phys. 35 (2009) 17-30.

[28] S.-H. Shim, M.T. Zanni, How to turn your pump-probe instrument into a multidimensional spectrometer: 2D IR and vis spectroscopies via pulse shaping, Phys. Chem. Chem. Phys. 11 (2009) 748-761.

[29] P.R. Griffiths, J.A. de Haseth, Fourier Transform Infrared Spectrometry, 2nd edition, Wiley, 2007.

[30] J.J. van Thor, K.L. Ronayne, M. Towrie, J.T. Sage, Balance between ultrafast parallel reactions in the green fluorescent protein has a structural origin, Biophys. J. 95 (2008) 1902-1912.

[31] R. Barer, A.R.H. Cole, H.W. Thompson, Infrared spectroscopy with the reflecting microscope in physics, chemistry and biology, Nature 163 (1949) 198-201.

[32] M.J. Fraser, R.D.B. Fraser, Evidence on the structure of desoxyribonucleic acid from measurements with polarized infrared radiation, Nature 167 (1951) 761-762.

[33] R. Wegmann, Spectrographie dans l'infra-rouge. VIII. Les acides desoxy - et ribonucléiques du noyau, Acta Histochem. 4 (1957) 132-140.

[34] M. Riedl, Optical Design Fundamentals for Infrared Systems, Second edition, SPIE Press, Bellingham, WA, 2001.

[35] K. Nariai, H. Iwamoto, A variation of Schwarzschild telescope: golden section solution with two concentric spheres and its extension to finite distance solutions, Opt. Rev. 12 (2005) 190-195, doi:10.1007/s10043-005-0190-z.

[36] I.A. Artioukov, K.M. Krymski, Schwarzschild objective for soft X-rays, Opt. Eng. 39 (2000) 2163-2170.

[37] G.L. Carr, Resolution limits for infrared microspectroscopy explored with synchrotron radiation, Rev. Sci. Instr. 72 (2001) 1613-1619.

[38] K. Gerwert, B. Hess, H. Michel, S. Buchanan, FTIR studies on crystals of photosynthetic reaction centers, FEBS Letts. 232 (1988) 303-307.

[39] D. A. Moss, J. T. Sage, unpublished observations.

[40] R. Efremov, V.I. Gordeliy, J. Heberle, G. Büldt, Time-resolved microspectroscopy on a single crystal of bacteriorhodopsin reveals lattice-induced differences in the photocycle kinetics, Biophys. J. 91 (2006) 1441-1451.

[41] M.W. Makinen, R.A. Houtchens, W.S. Caughey, Structure of carboxymyoglobin in crystals and in solution, Proc. Natl Acad. Sci. USA 76 (1979) 6042-6046.

[42] W.T. Potter, R.A. Houtchens, W.S. Caughey, Crystallization-induced changes in protein structure observed by infrared spectroscopy of carbon monoxide liganded to human hemoglobins A and Zürich, J. Am. Chem. Soc. 107 (1985) 3350. 
[43] M. Tsubaki, K. Shinzawa, S. Yoshikawa, Effects of crystallization on the hemecarbon monoxide moiety of bovine heart cytochrome $c$ oxidase carbonyl, Biophys. J. 63 (1992) 1564-1571.

[44] J.Heberle, G. Büldt, E. Koglin, J.P. Rosenbusch, E.M. Landau, Assessing the functionality of a membrane protein in a three-dimensional crystal, J. Mol. Biol. 281 (1998) 587-592.

[45] G.U. Nienhaus, K. Chu, K. Jesse, Structural heterogeneity and ligand binding in carbonmonoxy myoglobin crystals at cryogenic temperatures, Biochemistry 37 (1998) 6819-6823.

[46] A. Xie, L. Kelemen, J. Hendriks, B.J. White, K.J. Hellingwerf, W.D. Hoff, Formation of a new buried charge drives a large-amplitude protein quake in photoreceptor activation, Biochemistry 40 (2001) 1510-1517.

[47] J.-P. Colletier, D. Bourgeois, B. Sanson, D. Fournier, J.L. Sussman, I. Silman, M. Weik, Shoot-and-trap: use of specific X-ray damage to study structural protein dynamics by temperature-controlled cryo-crystallography, Proc. Natl Acad. Sci. USA 105 (2008) 11742-11747.

[48] J.O. Alben, G.H. Bare, P.A. Bromberg, Sulphydryl groups as a new molecular probe at the $\alpha_{1} \beta_{1}$ interface in haemoglobin using Fourier transform infrared spectroscopy, Nature 252 (1974) 736-738.

[49] Y. Sato, M. Nabeno, T. Iwata, S. Tokutomi, M. Sakurai, H. Kandori, Heterogeneous environment of the $\mathrm{S}-\mathrm{H}$ group of Cys966 near the flavin chromophore in the LOV2 domain of Adiantum neochrome1, Biochemistry 46 (2007) 10258-10265.

[50] M. Koziński, S. Garrett-Roe, P. Hamm, 2D-IR spectroscopy of the sulfhydryl band of cysteines in the hydrophobic core of proteins, J. Phys. Chem. B 112 (2008) 7645-7650.

[51] J.O. Alben, G.H. Bare, Ligand-dependent heme-protein interactions in human hemoglobin studied by Fourier transform infrared spectroscopy. Effects of quaternary structure on a chain tertiary structure measured at the $\alpha-104(\mathrm{G} 11)$ cysteine-SH, J. Biol. Chem. 255 (1980) 3892-3897.

[52] H. Khachfe, M. Mylrajan, J.T. Sage, Infrared crystallographic investigation of Tstate hemoglobin, Cell. Mol. Biol. (Noisy-le-grand) 44 (1998) 39-52.

[53] J.K. Chin, R. Jimenez, F.E. Romesberg, Protein dynamics and cytochrome c: correlations between ligand vibrations and redox activity, J. Am. Chem. Soc. 124 (2002) 1846-1847.

[54] M.E. Cremeens, J. Zimmermann, W. Yu, P.E. Dawson, F.E. Romesberg, Direct observation of structural heterogeneity in a $\beta$-sheet, J. Am. Chem. Soc. 131 (2009) 5726-5727.

[55] A. Dong, W.S. Caughey, Infrared methods for study of hemoglobin reactions and structures, Meth. Enzymol. 232 (1994) 139-175.

[56] R.P. Happe, W. Roseboom, A.J. Pierik, S.P. Albracht, K.A. Bagley, Biological activation of hydrogen, Nature 385 (1997) 126.

[57] L.J. Young, W.S. Caughey, Oxygenation of carbon monoxide by bovine heart cytochrome $c$ oxidase, Biochemistry 25 (1986) 152-161.

[58] V. Sampath, X.J. Zhao, W.S. Caughey, Anesthetic-like interactions of nitric oxide with albumin and hemeproteins. A mechanism for control of protein function, J. Biol. Chem. 276 (2001) 13635-13643.

[59] W. Mäntele, Reaction-induced infrared difference spectroscopy for the study of protein function and reaction mechanisms, Trends Biochem. Sci. 18 (1993) 197-202.

[60] A. Barth, Infrared spectroscopy of proteins, Biochim. Biophys. Acta 1767 (2007) 1073-1101.

[61] D. Marsh, Infrared dichroism of isotope-edited $\alpha$-helices and $\beta$-sheets, J. Mol. Biol. 338 (2004) 353-367.

[62] J.T. Sage, Infrared crystallography: structural refinement through spectroscopy, Appl. Spectrosc. 51 (1997) 568-573.

[63] J.T. Sage, Myoglobin and CO: structure, energetics, and disorder, J. Biol. Inorg. Chem. 2 (1997) 537-543, doi:10.1007/s007750050168.

[64] J.T. Sage, W. Jee, Structural characterization of the myoglobin active site using infrared crystallography, J. Mol. Biol. 274 (1997) 21-26.

[65] K.D. Rector, A.S. Kwok, C. Ferrante, A. Tokmakoff, C.W. Rella, M.D. Fayer, Vibrational anharmonicity and multilevel vibrational dephasing from vibrational echo beats, J. Chem. Phys. 106 (1997) 10027-10036.

[66] G.S. Kachalova, A.N. Popov, H.D. Bartunik, A steric mechanism for inhibition of CO binding to heme proteins, Science 284 (1999) 473-476.

[67] H. Kandori, Role of internal water molecules in bacteriorhodopsin, Biochim. Biophys. Acta 1460 (2000) 177-191.

[68] M. Born, E. Wolf, Principles of Optics: Electromagnetic Theory of Propagation, Interference and Diffraction of Light, Cambridge University Press, 1999.

[69] J.M. Stone, Radiation and Optics: An Introduction to the Classical Theory, McGraw-Hill, 1963.

[70] W.A. Eaton, T.P. Lewis, Polarized single crystal absorption spectrum of 1methyluracil, J. Chem. Phys. 53 (1970) 2164-2172.

[71] E. Hecht, Optics, 4th edition, Addison Wesley, 2002.

[72] J.W. Rohleder, T. Luty, The "oriented gas model" and its application to the infrared spectroscopy of molecular crystals, Mol. Cryst. 5 (1968) 145-163.

[73] T.A. Keiderling, Protein and peptide secondary structure and conformational determination with vibrational circular dichroism, Curr. Opin. Chem. Biol. 6 (2002) 682-688.

[74] J.T. Sage, D. Morikis, P.M. Champion, Resonance Raman studies of oriented chromophores: metmyoglobin single crystals, J. Chem. Phys. 90 (1989) 3015.

[75] D. Ivanov, J.T. Sage, M. Keim, J.R. Powell, S.A. Asher, P.M. Champion, Determination of CO orientation in myoglobin by single-crystal infrared linear dichroism, J. Am. Chem. Soc. 116 (1994) 4139-4140.

[76] F. Yang, G.N. Phillips Jr., Crystal structures of CO-, deoxy-, and met-myoglobins at various pH values, J. Mol. Biol. 256 (1996) 762-774.

[77] L. Zhu, J.T. Sage, A.A. Rigos, D. Morikis, P.M. Champion, Conformational interconversion in protein crystals, J. Mol. Biol. 224 (1992) 207-215.
[78] C.W. Rella, K.D. Rector, A. Kwok, J.R. Hill, H.A. Schwettman, D.D. Dlott, M.D. Fayer, Vibrational echo studies of myoglobin-CO, J. Phys. Chem. 100 (1996) 15620-15629.

[79] J. Berendzen, D. Braunstein, Temperature-derivative spectroscopy: a tool for protein dynamics, Proc. Natl Acad. Sci. USA 87 (1990) 1-5.

[80] A.M. Massari, I.J. Finkelstein, M.D. Fayer, Dynamics of proteins encapsulated in silica sol-gel glasses studied with IR vibrational echo spectroscopy, J. Am. Chem. Soc. 128 (2006) 3990-3997.

[81] R. Liddington, Z. Derewenda, G. Dodson, D. Harris, Structure of the liganded T state of haemoglobin identifies the origin of cooperative oxygen binding, Nature 331 (1988) 725-728.

[82] A. Mozzarelli, C. Rivetti, G.L. Rossi, E.R. Henry, W.A. Eaton, Crystals of haemoglobin with the $\mathrm{T}$ quaternary structure bind oxygen noncooperatively with no Bohr effect, Nature 351 (1991) 416-419.

[83] W. Mäntele, E. Nabedryk, B. Tavitian, W. Kreutz, J. Breton, Light-induced Fourie transform infrared (FTIR) spectroscopic investigations of the primary donor oxidation in bacterial photosynthesis, FEBS Letts. 187 (1985) 227-232.

[84] R. Moukhametzianov, J.P. Klare, R. Efremov, C. Baeken, A. Göppner, J. Labahn, M. Engelhard, G. Büldt, V.I. Gordeliy, Development of the signal in sensory rhodopsin and its transfer to the cognate transducer, Nature 440 (2006) 115-119.

[85] W. Gmelin, K. Zeth, R. Efremov, J. Heberle, J. Tittor, D. Oesterhelt, The crysta structure of the $\mathrm{L} 1$ intermediate of halorhodopsin at $1.9 \AA$ resolution, Photochem. Photobiol. 83 (2007) 369-377.

[86] U.K. Genick, G.E. Borgstahl, K. Ng, Z. Ren, C. Pradervand, P.M. Burke, V. Srajer, T.Y. Teng, W. Schildkamp, D.E. McRee, K. Moffat, E.D. Getzoff, Structure of a protein photocycle intermediate by millisecond time-resolved crystallography, Science 275 (1997) 1471-1475.

[87] H. Ihee, S. Rajagopal, V. Srajer, R. Pahl, S. Anderson, M. Schmidt, F. Schotte, P.A Anfinrud, M. Wulff, K. Moffat, Visualizing reaction pathways in photoactive yellow protein from nanoseconds to seconds, Proc. Natl Acad. Sci. USA 102 (2005) 7145-7150.

[88] V. Srajer, Z. Ren, T.Y. Teng, M. Schmidt, T. Ursby, D. Bourgeois, C. Pradervand, W. Schildkamp, M. Wulff, K. Moffat, Protein conformational relaxation and ligand migration in myoglobin: a nanosecond to millisecond molecular movie from time-resolved Laue X-ray diffraction, Biochemistry 40 (2001) 13802-13815.

[89] W.D. Tian, J.T. Sage, P.M. Champion, E. Chien, S.G. Sligar, Probing heme protein conformational equilibration rates with kinetic selection, Biochemistry 35 (1996) 3487-3502

[90] R.B.G. Ravelli, E.F. Garman, Radiation damage in macromolecular cryocrystallography, Curr. Opin. Struct. Biol. 16 (2006) 624-629.

[91] R.L. Owen, E. Rudiño-Piñera, E.F. Garman, Experimental determination of the radiation dose limit for cryocooled protein crystals, Proc. Natl Acad. Sci. USA 103 (2006) 4912-4917.

[92] A. Meents, S. Gutmann, A. Wagner, C. Schulze-Briese, Origin and temperature dependence of radiation damage in biological samples at cryogenic temperatures, Proc. Natl Acad. Sci. USA 107 (2010) 1094-1099.

[93] J.D. Watson, F.H. Crick, Molecular structure of nucleic acids: a structure for deoxyribose nucleic acid, Nature 171 (1953) 737-738.

[94] R.D.B. Fraser, The structure of deoxyribose nucleic acid, J. Struct. Biol. 145 (2004) 184-185.

[95] R.D.B. Fraser, T.P. MacRae, Conformation in Fibrous Proteins and Related Synthetic Polypeptides, Academic Press, New York, 1973.

[96] K.J. Rothschild, N.A. Clark, Polarized infrared spectroscopy of oriented purple membrane, Biophys. J. 25 (1979) 473-487.

[97] M.S. Braiman, K.J. Rothschild, Fourier transform infrared techniques for probing membrane protein structure, Annu. Rev. Biophys. Biophys. Chem. 17 (1988) 541-570.

[98] A. Ansari, C.M. Jones, E.R. Henry, J. Hofrichter, W.A. Eaton, Photoselection in polarized photolysis experiments on heme proteins, Biophys. J. 64 (1993) 852-868.

[99] M. Lim, T.A. Jackson, P.A. Anfinrud, Binding of CO to myoglobin from a heme pocket docking site to form nearly linear Fe-C-O, Science 269 (1995) 962-966

[100] M. Lim, T.A. Jackson, P.A. Anfinrud, Orientational distribution of CO before and after photolysis of $\mathrm{MbCO}$ and $\mathrm{HbCO}$ : a determination using time-resolved polarized mid-IR spectroscopy, J. Am. Chem. Soc. 126 (2004) 7946-7957.

[101] R.H. Havlin, N. Godbout, R. Salzmann, M. Wojdelski, W. Arnold, C.E. Schulz, E. Oldfield, An experimental and density functional theoretical investigation of iron-57 Mössbauer quadrupole splittings in organometallic and heme-model compounds: applications to carbonmonoxy-heme protein structure, J. Am. Chem. Soc. 120 (1998) 3144-3151.

[102] J. Vojtechovský, K. Chu, J. Berendzen, R.M. Sweet, I. Schlichting, Crystal structures of myoglobin-ligand complexes at near-atomic resolution, Biophys. J. 77 (1999) 2153-2174.

[103] B. Stec, G.N. Phillips, How the CO in myoglobin acquired its bend: lessons in interpretation of crystallographic data, Acta Cryst. D 57 (2001) 751-754.

[104] T.G. Spiro, P.M. Kozlowski, Discordant results on FeCO deformability in heme proteins reconciled by density functional theory, J. Am. Chem. Soc. 120 (1998) 4524-4525.

[105] W.R. Scheidt, A. Barabanschikov, J.W. Pavlik, N.J. Silvernail, J.T. Sage, Electronic structure and dynamics of nitrosyl porphyrins, Inorg. Chem. 49 (2010) 6240-6252.

[106] T.G. Spiro, P.M. Kozlowski, Is the CO adduct of myoglobin bent, and does it matter? Acc. Chem. Res. 34 (2001) 137-144.

[107] M. Unno, J.F. Christian, J.S. Olson, J.T. Sage, P.M. Champion, Evidence for hydrogen bonding effects in the iron ligand vibrations of carbonmonoxy myoglobin, J. Am. Chem. Soc. 120 (1998) 2670-2671

[108] L.M. Gregoret, S.D. Rader, R.J. Fletterick, F.E. Cohen, Hydrogen bonds involving sulfur atoms in proteins, Proteins 9 (1991) 99-107. 
[109] A. Mozzarelli, C. Rivetti, G.L. Rossi, W.A. Eaton, E.R. Henry, Allosteric effectors do not alter the oxygen affinity of hemoglobin crystals, Protein Sci. 6 (1997) 484-489.

[110] D.M. Wieliczka, S. Weng, M.R. Querry, Wedge shaped cell for highly absorbent liquids: Infrared optical constants of water, Appl. Opt. 28 (1989) 1714-1719.

[111] Photosystem II from Thermosynechococcus elongatus crystallized in space group $P 2_{1} 2_{1} 2_{1}$ using hanging drop vapor diffusion against a reservoir containing $8-14 \%$ PEG-4000, 20\% glycerol, 100 mM HEPES pH 7.5, 100 mM ammonium sulfate, and $0.11 \mathrm{mM}$ polyoxyethylene lauryl ether (C12E8) [112]. The solution sample was $8.4 \mathrm{mg}$ chlorophyll/mL in $20 \mathrm{mM}$ MES pH 6.5, $10 \mathrm{mM} \mathrm{CaCl} 2$ and $10 \mathrm{mM} \mathrm{MgCl}$, with $1 \mathrm{mM}$ DCMU (3-(3,4-dichlorophenyl)-1,1-dimethylurea). Excess n-dodecyl $\beta$-D-maltoside (DDM), used to solubilize photosystem II, was removed during anion exchange purification.

[112] K.N. Ferreira, T.M. Iverson, K. Maghlaoui, J. Barber, S. Iwata, Architecture of the photosynthetic oxygen-evolving center, Science 303 (2004) 1831-1838.

[113] BChE was purified and crystallized as described [115]. Crystals were transferred for $10 \mathrm{~s}$ into a cryoprotectant solution containing $2.3 \mathrm{M}$ ammonium sulfate $0.1 \mathrm{M}$ morpholinoethanesulfonic acid (MES), $\mathrm{pH} 6.5$, and $16 \%$ glycerol. Subsequently, they were loop-mounted and flash-cooled in a $100 \mathrm{~K}$ nitrogen gas stream from a commercial cryocooler (600 series, Oxford Cryosystems, Oxford, UK). Control experiments were performed on a thin film of cryoprotectant flash-cooled on a loop in the cryostream at $100 \mathrm{~K}$. Crystals and cryoprotectant were irradiated for the indicated exposure times at beamline ID14-EH4 at the European Synchrotron Radiation Facility (ESRF) [116], using the unattenuated beam $(300 \mu \mathrm{m} \times 300 \mu \mathrm{m})$ at an energy of $13.2 \mathrm{keV}$. Subsequent to irradiation, samples were retrieved in liquid nitrogen and shipped at $77 \mathrm{~K}$ to Northeastern University, where data were recorded using an infrared microscope. Both irradiation and IR measurements were performed with the cryoloops mounted in a $100 \mathrm{~K}$ cryostream.

[114] K.S. Paithankar, E.F. Garman, Know your dose: RADDOSE, Acta Cryst. D 66 (2010) 38-388.

[115] Y. Nicolet, O. Lockridge, P. Masson, J.C. Fontecilla-Camps, F. Nachon, Crystal structure of human butyrylcholinesterase and of its complexes with substrate and products, J. Biol. Chem. 278 (2003) 41141-41147.

[116] A.A. McCarthy, S. Brockhauser, D. Nurizzo, P. Theveneau, T. Mairs, D. Spruce, M. Guijarro, M. Lesourd, R.B.G. Ravelli, S. McSweeney, A decade of user operation on the macromolecular crystallography MAD beamline ID14-4 at the ESRF, J. Synchrotron Radiat. 16 (2009) 803-812.

[117] M.T. Facciotti, S. Rouhani, F.T. Burkard, F.M. Betancourt, K.H. Downing, R.B. Rose, G. McDermott, R.M. Glaeser, Structure of an early intermediate in the M-state phase of the bacteriorhodopsin photocycle, Biophys. J. 81 (2001) 3442-3455.

[118] F.M. Hendrickson, F. Burkard, R.M. Glaeser, Structural characterization of the L-to-M transition of the bacteriorhodopsin photocycle, Biophys. J. 75 (1998) 1446-1454. 